\title{
Impacto de los cambios demográficos en las demandas sectoriales en América Latina*
}

\author{
Paulo Murad Saad** \\ Tim Miller*** \\ Ciro Martínez****
}

\begin{abstract}
América Latina se encuentra actualmente en un proceso de transición demográfica que afecta profundamente la distribución por edades de la población. Dado que el comportamiento económico de las personas varía según la etapa del ciclo de vida en la que se encuentran, los cambios en la estructura por edades tienden a producir un impacto importante sobre el proceso de desarrollo económico. En base a patrones etarios de consumo e ingresos laborales construidos a partir de los datos del proyecto Cepal / IDRC sobre transferencias intergeneracionales, el artículo trata de examinar el impacto económico de los cambios demográficos, particularmente del bono demográfico, en tres sectores directamente relacionados con el bienestar de la población y el desarrollo económico de la región: la educación, la salud y el sistema de reparto de pensiones. El análisis revela que, en el ámbito educativo, se prevé un aumento sustancial de los recursos disponibles para el próximo decenio, lo cual permitiría sostener económicamente los esfuerzos encaminados a ampliar la cobertura en los distintos niveles educacionales. En el ámbito de la salud, mientras en algunos países se prevé un aumento de los recursos disponibles por consumidor, en aquellos más avanzados en la transición demográfica, se proyecta más bien una desventaja demográfica para el próximo decenio. En gran contraste con el caso de la educación, el cambio demográfico impone mayores dificultades para el financiamiento de los sistemas de reparto de pensiones. El efecto del envejecimiento de la población en estos sistemas es evidente en casi todos los países de la región.
\end{abstract}

Palabras Clave: bono demográfico, envejecimiento, sectores sociales.

\footnotetext{
* Este artigo está baseado no relatório "Transformações demográficas e sua influência sobre o desenvolvimento na América Latina e no Caribe" (CEPAL, 2008), apresentado ao Comitê Especial de População e Desenvolvimento durante ○ 32 o período de sessões da Cepal, realizado em Santo Domingo, República Dominicana, de 9 a 13 de junho de 2008. 0 relatório foi elaborado sob direção e coordenação de Dirk Jaspers, diretor do Centro Latino Americano e Caribenho de Demografia (Celade) - Divisão de População da Cepal, e contou com o apoio do Fundo de População das Nações Unidas (UNFPA). A redação esteve a cargo de Susana Schkolnik, com a colaboração de Paulo Saad e Tim Miller. Também contou com a colaboração de Sandra Huenchuan, Ciro Martínez, Daniela González, Juan Chackiel, Guiomar Bay e Mauricio Holz. ** Ph.D. em Sociologia, Universidade do Texas, Austin, oficial de Assuntos Populacionais, Centro Latino Americano e Caribenho de Demografia (Celade) - Divisão de População da Comissão Econômica para América Latina e o Caribe (Cepal), das Nações Unidas.

Ph.D. em Demografia, Universidade da Califórnia, Berkeley, oficial de Assuntos Populacionais, Centro Latino Americano e Caribenho de Demografia (Celade) - Divisão de População da Comissão Econômica para América Latina e o Caribe (Cepal), das Nações Unidas.

${ }_{* \star \star \star}$ Ph.D. em Demografia, Universidade Autônoma de Barcelona, consultor, Centro Latino Americano e Caribenho de Demografia (Celade) - Divisão de População da Comissão Econômica para América Latina e o Caribe (Cepal), das Nações Unidas.
} 


\section{Introducción}

Al igual que otras regiones en desarroIlo, América Latina se encuentra actualmente en un proceso de transición demográfica, en el que se parte de una situación inicial de altas tasas de mortalidad y fecundidad para arribar a un escenario final de tasas significativamente más reducidas. Tal transición afecta profundamente la distribución por edades de la población, en especial porque la reducción de las tasas de mortalidad y fecundidad no se produce de manera simultánea. Por lo general, la reducción de la mortalidad antecede en algunas décadas a la de la natalidad, produciendo un "boom" generacional durante el periodo en que la mortalidad, principalmente la infantil, se reduce, mientras que la fecundidad aún permanece elevada. En esta fase inicial de la transición demográfica, el control de la mortalidad infantil y de la niñez produce un mayor crecimiento de la población, además de su rejuvenecimiento como consecuencia del aumento de la proporción de niños. Con el descenso de la fecundidad, el crecimiento poblacional disminuye su ritmo, y en la medida en que las generaciones más numerosas avanzan en el ciclo de vida, se produce un progresivo envejecimiento en la estructura etaria de la población.

Dado que el comportamiento económico de las personas varía según la etapa del ciclo de vida en la que se encuentran, los cambios en la estructura por edades tienden a producir un impacto importante sobre el proceso de desarrollo económico. Una alta proporción de personas dependientes, niños o adultos mayores, tiende a limitar el crecimiento económico, ya que una parcela significativa de los recursos se destina a atender sus demandas. Por el contrario, una alta proporción de personas en edad de trabajar puede impulsar el crecimiento económico, a través del incremento del ingreso y la acumulación acelerada del capital resultante del mayor porcentaje de trabajadores, y de la reducción del gasto en las personas dependientes.

Durante la transición demográfica hay un periodo en el que la proporción de personas en edades económicamente activas crece de manera sostenida en relación a la de personas en edades dependientes, particularmente de niños. De ahí surge el concepto de "bono demográfico", el cual supone que el aumento de la población en edades activas, respecto a aquella en edades dependientes, podrá apoyar el despegue económico y contribuir a mitigar la carga económica que esta última implica.

Aunque el período del bono demográfico pueda durar varias décadas, la ventaja de una relación favorable entre la población en edades activas y dependientes se modificará eventualmente, como resultado del incremento continuo de la proporción de adultos mayores. Este período de rápido envejecimiento poblacional trae aparejados nuevos desafíos para la sociedad, que debe adaptarse a una situación sin precedentes, y requiere el desarrollo de políticas públicas que faciliten los ajustes necesarios en diversas áreas, incluyendo la provisión de cuidados de salud y el apoyo a la población adulta mayor. La necesidad de tales ajustes da margen para el planteamiento de cuestiones sobre equidad intergeneracional en el cumplimiento de las necesidades de las personas en diferentes etapas del ciclo de vida, y sobre el papel que juegan en este proceso el Estado, el sector privado y la familia. De ahí la importancia de aprovechar el bono demográfico, incluso como una forma de prepararse mejor para el desafío que plantea el envejecimiento.

Este artículo tiene por objetivo examinar el impacto económico de los cambios demográficos, particularmente del bono demográfico, en tres sectores directamente relacionados con el bienestar de la población y el desarrollo económico de los países de América Latina: la educación, la salud y el sistema de reparto de pensiones. En la primera parte se analizan brevemente las tendencias demográficas pasadas, presentes y futuras en América Latina, en base a las estimaciones y proyecciones de población producidas por el Centro Latinoamericano y Caribeño de Demografía (Celade) -División de Población de la Comisión Económica para América Latina y el Caribe (Cepal)-. El análisis incluye aspectos relativos a la transición demográfica, los cambios en la relación 
de dependencia y el bono demográfico, buscando ilustrar la diversidad de situaciones demográficas que prevalece en la región. En la segunda parte se estiman los efectos económicos -totales y específicos en cada uno de los sectores sociales del bono demográfico, en base a patrones etarios de consumo e ingresos laborales construidos a partir de los datos del proyecto del Celade sobre transferencias intergeneracionales, envejecimiento de la población y protección social en América Latina. ${ }^{1}$

\section{Tendencias demográficas en América Latina}

\section{Transición demográfica}

Muchos estudios han tratado de analizar el proceso de transición demográfica en América Latina, en el que las tasas de mortalidad y fecundidad pasan de unos niveles bastante elevados a otros significativamente más reducidos (ZAVALA DE COSíO, 1995; WONG; CARVALHO; AGUIRRE, 2000; FÍGOLI; WONG, 2003; Cepal, 2008a). De una manera general, durante la fase inicial de la transición -cuando la mortalidad, principalmente la infantil, se redujo, mientras la fecundidad permanecía elevada, la población de la región se mantuvo considerablemente joven, y hasta se rejuveneció levemente a consecuencia del aumento proporcional de los niños. Desde mediados de los años sesenta, el descenso continuado de la fecundidad, aunado a un aumento sostenido de la esperanza de vida, dio inicio a un proceso de envejecimiento progresivo de la población, a medida que las generaciones más numerosas, nacidas durante el periodo inicial de la transición demográfica, fueron avanzando en el ciclo de vida.
La mayoría de los países de América Latina inició el proceso de transición demográfica a mediados de la década de 1960, y se encuentra transitando la fase de disminución de la fecundidad, que se ha producido en forma rápida, después de haber experimentado cambios importantes en la mortalidad. Sin embargo, este proceso no se ha presentado de manera uniforme en el interior de la región. Actualmente coexisten diversas realidades, asociadas con la etapa de la transición demográfica en la que se encuentran los países.

El cuadro 1 presenta un resumen de las transformaciones demográficas ocurridas en el pasado y proyectadas para el futuro en América Latina. Entre 1950 y la fecha actual, el número promedio de hijos de las mujeres latinoamericanas ha bajado de 5,9 a 2,4; las muertes de niños menores de 1 año se han reducido de 128 a 22 por cada mil nacimientos; la expectativa media de vida de la población ha crecido de 52 a 73 años, y la tasa media anual de crecimiento ha disminuido de $2,8 \%$ a $1,3 \%$. En el mismo periodo, la proporción de niños menores de 15 años se ha reducido de $40 \%$ a $30 \%$, mientras que la de personas de 60 años y más ha aumentado de $6 \%$ a $9 \%$. Se espera que hasta el año 2050 la esperanza de vida haya alcanzado los 80 años, mientras que el número promedio de hijos por mujer haya bajado a 1,9 (inferior, por lo tanto, a la tasa aproximada de reemplazo, de 2,1 hijos por mujer), la mortalidad infantil a 8 muertes por mil nacimientos y la tasa media de crecimiento poblacional a $0,2 \%$. Como resultado de tales cambios, se proyecta que en 2050 la proporción de niños menores de 15 años se habrá reducido a casi a la mitad (del $30 \%$ al $18 \%$ ), mientras que la de personas mayores se habrá casi triplicado, pasando del $9 \%$ al

\footnotetext{
1 El proyecto Transferencias intergeneracionales, envejecimiento de la población y protección social en América Latina (en línea: http://www.eclac.cl/celade/proyecto_transferencias) cuenta con el apoyo financiero del Centro Internacional de Investigaciones para el Desarrollo de Canadá (International Development Research Centre - IDRC, Project \# 104231), y es parte de un proyecto internacional sobre cuentas de transferencias nacionales (National Transfers Accounts - NTA, en línea: http://www.schemearts.com/proj/nta/web/nta/show/), coordinado por los profesores Ronald Lee (UC Berkeley) y Andrew Mason (University of Hawai), con el apoyo del Instituto Nacional de Salud de los Estados Unidos (US National Institutes of Health, grant NIA R01-AG25247). Se agradece a los investigadores de los cinco países que participan actualmente en el proyecto (Brasil, Chile, Costa Rica, México y Uruguay), quienes han producido parte de la información a nivel nacional utilizada para fines comparativos en este artículo.
} 
CUADRO 1

Indicadores de la dinámica demográfica

América Latina - 1950-2050

\begin{tabular}{lccccc}
\hline Indicadores & $\mathbf{1 9 5 0 - 1 9 5 5}$ & $\mathbf{1 9 7 5 - 1 9 8 0}$ & $\mathbf{2 0 0 5 - 2 0 1 0}$ & $\mathbf{2 0 2 5 - 2 0 3 0}$ & $\mathbf{2 0 4 5 - 2 0 5 0}$ \\
\hline $\begin{array}{l}\text { Tasa global de fecundidad } \\
\text { (hijos por mujer) }\end{array}$ & 5,9 & 4,5 & 2,4 & 2,0 & 1,9 \\
$\begin{array}{l}\text { Esperanza de vida } \\
\text { al nacer (años) }\end{array}$ & 51,8 & 63,4 & 73,4 & 77,1 & 79,6 \\
$\begin{array}{l}\text { Tasa de mortalidad infantil } \\
\text { (por mil nacimientos) }\end{array}$ & 127,7 & 69,7 & 21,7 & 12,0 & 7,9 \\
$\begin{array}{l}\text { Tasa anual de crecimiento } \\
\text { poblacional (\%) }\end{array}$ & 2,8 & 2,3 & 1,3 & 0,7 & 0,2 \\
Estructura etaria (\%) & 1950 & 1975 & 2005 & 2025 & 2050 \\
$\quad 0$ a 14 años & 40 & 41 & 30 & 23 & 18 \\
15 a 59 años & 54 & 52 & 61 & 62 & 58 \\
60 años y más & 6 & 6 & 9 & 14 & 24 \\
$\quad$ Total & 100 & 100 & 100 & 100 & 100 \\
\hline
\end{tabular}

Fuente: Centro Latinoamericano y Caribeño de Demografía (CELADE) - División de Población de la CEPAL, Estimaciones y proyecciones de población, 2007.

24\%. De ese modo, para la mitad de este siglo, uno de cada cuatro latinoamericanos será una persona mayor.

Sin embargo, los promedios regionales esconden situaciones muy disímiles entre los distintos países de América Latina en términos del ritmo y la intensidad de los cambios demográficos. Con el fin de analizar esta heterogeneidad, y de obtener una visión global de los cambios ocurridos en la región, la Cepal (2008a) clasificó a los países latinoamericanos en cuatro etapas distintas de la transición demográfica -muy avanzada, avanzada, plena y moderada, de acuerdo a sus niveles de fecundidad y esperanza de vida.

Cuba ha sido clasificada, de manera solitaria, en la etapa de transición muy avanzada, ya que su tasa global de fecundidad alcanzó el nivel de reemplazo muy precozmente en relación a los estándares regionales, entre fines de los años setenta y comienzos de los ochenta. Los países ubicados en la etapa de transición avanzada, por otro lado, se dividen en tres subgrupos. El primero, integrado por Argentina y Uruguay, se caracteriza por un descenso temprano de las tasas vitales, ya que en ambos se registraron transiciones tanto de la fecundidad como de la mortalidad en la primera mitad del siglo pasado, y ya a partir de 1950 exhibían niveles de fecundidad cercanos a los 3 hijos por mujer.

El segundo subgrupo está conformado únicamente por Chile, que si bien en el quinquenio 1950-1955 mostraba un nivel de fecundidad similar al de Cuba, tuvo un descenso menos acentuado. Aunque actualmente su tasa no es tan baja como la cubana, es el segundo país que ya se encuentra por debajo del nivel de reemplazo de su población. Tanto en Argentina y Uruguay como en Chile, las tasas actuales de crecimiento demográfico son inferiores al $1 \%$.

El tercer subgrupo dentro de la etapa de transición avanzada lo integran Brasil, Colombia, Costa Rica y México. Estos países, en contraste con los demás clasificados en esta fase, tenían tasas globales de fecundidad bastante elevadas hasta mediados de los años sesenta, pero avanzaron rápidamente en la transición de la fecundidad, de tal modo que esta se redujo a la mitad o menos hacia fines de los años ochenta y comienzos de los noventa. Sus tasas de crecimiento demográfico natural (que no consideran los movimientos migratorios) oscilan entre un $1,3 \%$ y un $1,4 \%$.

En la fase de transición plena coinciden dos grupos de países con trayectorias demográficas distintas. Por un lado están Ecuador, El Salvador, Panamá, Perú, la 
República Dominicana y la República Bolivariana de Venezuela, que habían logrado considerables descensos de la fecundidad a comienzos de los años ochenta, y por otro están Honduras, Nicaragua y Paraguay, donde las reducciones más significativas tuvieron lugar recientemente en los últimos años. En la actualidad, ambos grupos de países se han equiparado en un nivel de fecundidad intermedio dentro de la región, pero con tasas de crecimiento demográfico natural aún heterogéneas, y que van desde el $1,5 \%$ en Perú al $2,3 \%$ en Honduras.

Finalmente, en la etapa de transición moderada se encuentran el Estado Plurinacional de Bolivia, Haití y Guatemala, países en los que si bien la fecundidad ha bajado, sigue muy por encima del promedio regional. En Guatemala el descenso de la fecundidad ha sido particularmente lento, siendo su tasa de fecundidad la más alta de la región en la actualidad ( 4,2 hijos por mujer), al igual que su tasa de crecimiento demográfico natural (2,8\%). Haití y el Estado Plurinacional de Bolivia, por otro lado, presentan las tasas más altas de mortalidad de América Latina, lo que se traduce en esperanzas de vida muy por debajo del promedio regional (61 y 66 años, respectivamente, frente a 73 años para la región).

Pese a las persistentes diferencias en el interior de la región, se podría decir, de una manera general, que América Latina ha experimentado un proceso considerablemente más rápido de transición demográfica en comparación con la experiencia de los países actualmente industrializados (Cepal/ CELADE/BID, 1996). Mientras en estos últimos el proceso se extendió por más de una centuria, en América Latina apenas supera el medio siglo, en gran parte debido a que a su comienzo ya estaban disponibles los medios para un mejor control, tanto de la mortalidad como de la fecundidad. Como consecuencia, los cambios en la estructura por edades de la población, y en particular su envejecimiento, han sido mucho más rápidos en América Latina que en los países industrializados, lo que presupone la necesidad de medidas diferenciadas para hacer frente a los desafíos y aprovechar las oportunidades que se derivan de la dinámica demográfica.

\section{Cambios en la relación de dependencia}

Es común utilizar indicadores calculados sobre la base de rangos etarios para comparar la situación de los países, y evaluar la manera en que las condiciones demográficas se relacionan con otros aspectos del desarrollo socioeconómico. La relación de dependencia, por ejemplo, al vincular la población en edades potencialmente inactivas con aquella en edades potencialmente activas, constituye un importante indicador de los efectos potenciales de los cambios demográficos sobre el desarrollo socioeconómico.

Sin embargo, es importante tener en cuenta las limitaciones asociadas a las relaciones de dependencia expresadas en términos de rangos etarios. Por un lado, las personas no dejan de ser económicamente activas de manera automática a partir de una cierta edad, y no todas las personas en edades potencialmente activas son efectivamente activas en términos económicos (particularmente en el caso de las mujeres, pese su creciente participación laboral). Por otro lado, en la medida en que se incrementa la etapa de entrenamiento para la vida laboral, un número creciente de adolescentes y adultos jóvenes permanece más tiempo en la escuela y fuera del mercado de trabajo, extendiendo el periodo de dependencia joven. Estas observaciones sugieren que las tendencias en las relaciones de dependencia representan apenas un indicativo del impacto económico resultante de los cambios entre grupos etarios clave de la población.

En el presente estudio se consideró como potencialmente activo el grupo de entre 15 y 59 años, y como potencialmente inactivas a las personas menores de 15 años y a las de 60 años y más. En base a esta definición, se estima que la relación de dependencia promedio en América Latina experimentó un aumento entre 1950 y mediados de los años sesenta, debido a un incremento relativo de la población infantil, y a partir de entonces empezó a reducirse de manera sostenida, a causa de la caída 
de la fecundidad. Se proyecta que esta reducción continúe hasta aproximadamente el año 2020, cuando la relación vuelva a crecer gradualmente, a causa del incremento relativo de la población adulta mayor. Aunque de una manera general los países latinoamericanos se ajustan a este modelo en cuanto a su forma, existe una variación enorme entre ellos en términos de timing (véase el cuadro 2).

El gráfico 1 ilustra la diversidad de trayectorias que seguirá la relación de dependencia en las próximas décadas, de acuerdo a la etapa de la transición demográfica en que se encuentran los países de la región. El gráfico toma el año 2008 como base (igual a 100), contra la que compara los valores proyectados hasta 2050 . Se observa claramente una tendencia decreciente de la relación de dependencia en los países más rezagados en el proceso de transición, como el Estado Plurinacional de Bolivia y Guatemala, y otra fuertemente ascendente en el caso de aquellos más avanzados en el proceso, como Cuba y Chile. Mientras tanto, en países como Brasil y México la relación de dependencia tiende a crecer de manera similar al promedio latinoamericano, o sea, con una intensidad significativamente menor que en los países en etapas más avanzadas.

CUADRO 2

Aspectos relativos a la evolución de la relación de dependencia (1) (RD) Países de América Latina

\begin{tabular}{|c|c|c|c|c|c|c|c|c|c|}
\hline \multirow[b]{2}{*}{ Países } & \multicolumn{6}{|c|}{ Período de reducción de la RD } & \multicolumn{3}{|c|}{$\begin{array}{c}\text { Período en que la RD se } \\
\text { mantiene inferior a } 2 / 3\end{array}$} \\
\hline & $\begin{array}{c}\text { Valor } \\
\text { máximo } \\
\text { (VMA) }\end{array}$ & $\begin{array}{l}\text { Año del } \\
\text { VMA }\end{array}$ & $\begin{array}{l}\text { Valor } \\
\text { mínimo } \\
\text { (VMI) }\end{array}$ & $\begin{array}{l}\text { Año del } \\
\text { VMI }\end{array}$ & $\begin{array}{l}\text { Duración } \\
\text { (años) }\end{array}$ & $\begin{array}{l}\text { Magnitud } \\
(\%)\end{array}$ & Año inicial & Año final & $\begin{array}{l}\text { Duración } \\
\text { (años) }\end{array}$ \\
\hline América Latina & 97 & 1965 & 60 & 2019 & 54 & 38 & 2001 & 2041 & 40 \\
\hline Argentina & 78 & 1989 & 63 & 2032 & 43 & 19 & 2011 & 2037 & 26 \\
\hline Bolivia (E. P. de) & 95 & 1974 & 57 & 2041 & 67 & 40 & 2021 & 2060 & 39 \\
\hline Brasil & 97 & 1964 & 58 & 2007 & 43 & 41 & 1996 & 2038 & 42 \\
\hline Chile & 92 & 1966 & 54 & 2011 & 45 & 41 & 1985 & 2024 & 39 \\
\hline Colombia & 109 & 1965 & 56 & 2017 & 52 & 49 & 2000 & 2039 & 39 \\
\hline Costa Rica & 115 & 1965 & 53 & 2014 & 49 & 54 & 2000 & 2038 & 38 \\
\hline Cuba & 91 & 1974 & 53 & 1991 & 17 & 42 & 1983 & 2024 & 41 \\
\hline Ecuador & 105 & 1965 & 61 & 2025 & 60 & 43 & 2010 & 2045 & 35 \\
\hline El Salvador & 104 & 1968 & 57 & 2028 & 60 & 45 & 2013 & 2047 & 34 \\
\hline Guatemala & 103 & 1988 & 55 & 2050 & 62 & 46 & 2031 & 2067 & 36 \\
\hline Haití & 92 & 1970 & 57 & 2039 & 69 & 38 & 2021 & 2057 & 36 \\
\hline Honduras & 111 & 1972 & 56 & 2040 & 68 & 50 & 2021 & 2057 & 36 \\
\hline México & 110 & 1966 & 57 & 2022 & 56 & 48 & 2005 & 2034 & 29 \\
\hline Nicaragua & 114 & 1965 & 59 & 2035 & 70 & 48 & 2015 & 2050 & 35 \\
\hline Panamá & 102 & 1968 & 61 & 2020 & 52 & 40 & 2003 & 2031 & 28 \\
\hline Paraguay & 113 & 1962 & 58 & 2038 & 76 & 48 & 2018 & 2053 & 35 \\
\hline Perú & 99 & 1967 & 59 & 2017 & 50 & 41 & 2006 & 2046 & 40 \\
\hline Rep. Dominicana & 114 & 1965 & 63 & 2027 & 62 & 44 & 2015 & 2042 & 27 \\
\hline Uruguay (2) & 74 & 1989 & 67 & 2016 & 27 & 9 & - & - & - \\
\hline $\begin{array}{l}\text { Venezuela } \\
\text { (Rep. Bol.) }\end{array}$ & 104 & 1966 & 61 & 2020 & 54 & 41 & 2003 & 2045 & 42 \\
\hline
\end{tabular}

Fuente: Centro Latinoamericano y Caribeño de Demografía (CELADE) - División de Población de la CEPAL, Estimaciones y proyecciones de población, 2007.

(1) Número de personas menores de 15 años y de 60 años y más por cada cien personas de entre 15 y 59 años.

(2) Después de alcanzar su valor máximo, la relación de dependencia en Uruguay ya no deciende por debajo de 2/3. 
GRÁFICO 1

Tendencia futura de la relación de dependencia total (1)

Países seleccionados de América Latina - 2008-2050

$(2008=100)$

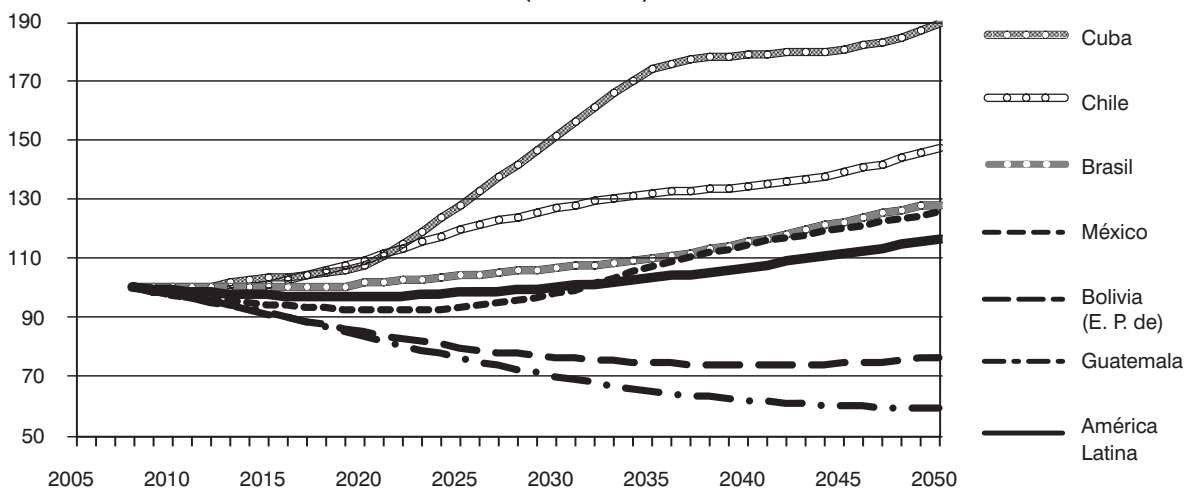

Fuente: Centro Latinoamericano y Caribeño de Demografía (CELADE) - División de Población de la CEPAL, Estimaciones y proyecciones de población, 2007

(1) Número de personas menores de 15 años y de 60 años y más por cada cien personas de entre 15 y 59 años.

La comparación entre los casos extremos de Cuba y Guatemala da una buena idea de la diversidad de situaciones demográficas que coexisten en la región: mientras en Cuba se espera que la relación de dependencia prácticamente se duplique hasta el año 2050, en Guatemala se proyecta que la relación se reducirá casi a la mitad en el mismo periodo.

\section{Bono demográfico}

En general, se define como "bono demográfico" o "ventana demográfica de oportunidades" al periodo durante la transición demográfica en que la relación de dependencia disminuye, o sea, en que aumenta el peso de las personas en edades potencialmente productivas, en relación con el de aquellas en edades mayormente inactivas (BLOOM; CANNING; SEVILLA, 2003; WONG; CARVALHO, 2006a). En este periodo se crea un contexto especialmente favorable para el desarrollo, debido a la mayor viabilidad del ahorro y la oportunidad de inversiones en el crecimiento económico, al mismo tiempo en que se reduce la presión por recursos en la educación básica.

Existe un considerable consenso respecto a que, si bien el bono demográfico ofrece una oportunidad para el crecimiento económico, la realización de estos beneficios depende de la adopción de políticas ma- croeconómicas que incentiven la inversión productiva, aumenten las oportunidades de empleo y promuevan un ambiente social y económico estable, propicio para un desarrollo sostenido (ADIOETOMO y otros, 2005; BLOOM; CANNING; SEVILLA, 2006; WONG; CARVALHO, 2006b). En particular, se requieren fuertes inversiones en capital humano, especialmente en lo que se refiere a la educación de los jóvenes, a fin de que las generaciones cuantitativamente mayores sean también cualitativamente más productivas.

De hecho, la combinación de una fuerza de trabajo amplia, joven y altamente capacitada para el empleo, y un contingente de personas mayores dependientes relativamente pequeño, constituye una situación altamente favorable para el crecimiento económico, como lo demuestra la historia reciente de los países del sudeste asiático. Bloom y Williamson (1998), por ejemplo, estiman que el aumento de la proporción de trabajadores, y su consecuente efecto sobre el ahorro, están asociados a un tercio del $6 \%$ promedio anual que registró el crecimiento de estas economías entre 1965 y 1990 . El aumento en la productividad observado en estos países se debió en gran medida a las fuertes inversiones en la educación de los jóvenes y a la ampliación de las oportunidades de empleo, por medio de cambios en la estructura industrial y ocupacional, durante 
el período del bono demográfico (UNFPA, 1998; MASON, 2002).

En general, no existe una medida exacta de los límites del bono demográfico, y su definición en términos de la evolución de la relación de dependencia suele variar. Por lo general, en su fase decreciente, la relación de dependencia en América Latina se mantiene aún extremadamente elevada durante un cierto período de tiempo, y después de alcanzar su valor mínimo tiende a permanecer relativamente baja por un determinado lapso, antes de volver a niveles más altos. Para efectos de ilustración, en el presente artículo se divide el periodo del bono demográfico en tres fases distintas: en la primera (bono 1) la relación de dependencia disminuye, pero todavía se mantiene relativamente alta, por encima de dos personas dependientes por cada tres en edades activas. En la segunda fase (bono 2) alcanza niveles más favorables, por debajo de dos personas dependientes para cada tres en edades activas, y sigue bajando. Finalmente, en la tercera fase (bono 3), la relación de dependencia empieza a subir, debido al aumento proporcional de las personas mayores, pero todavía se mantiene en niveles favorables, por debajo de dos personas dependientes por cada tres en edades activas. ${ }^{2}$

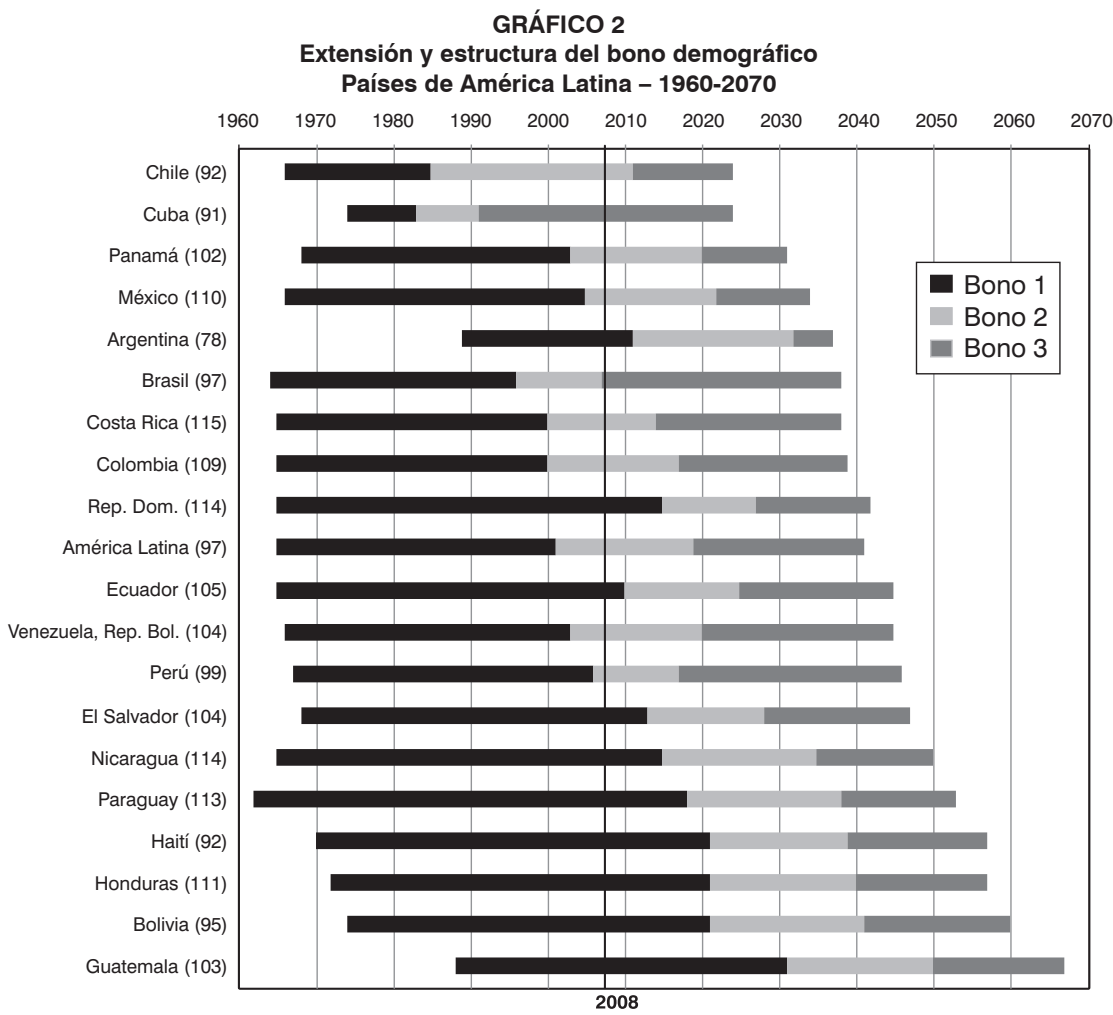

Fuente: Centro Latinoamericano y Caribeño de Demografía (Celade) - División de Población de la Cepal, Estimaciones y proyecciones de población, 2007.

Nota: Bono 1: periodo en que relación de dependencia es decreciente pero mayor que 2/3, Bono 2: periodo en que relación de dependencia es decreciente y menor que $2 / 3$, Bono 3 : periodo en que relación de dependencia es creciente pero menor que $2 / 3$ Los números entre paréntesis, al lado de los nombres de países, se refieren al valor de la relación de dependencia al inicio del bono demográfico.

El gráfico no incluye a Uruguay debido a que después de alcanzar su valor máximo, la relación de dependencia en este país ya no desciende por debajo de 2 personas dependientes para cada 3 en edades activas.

\footnotetext{
2 Dos personas dependientes por cada tres en edades activas corresponde a un valor aproximadamente $10 \%$ superior al valor mínimo de la relación de dependencia promedio de América Latina.
} 
Aunque el bono demográfico, conforme fue definido arriba, no varía de manera significativa entre los países de la región -en casi todos, la relación de dependencia empieza a bajar alrededor de la mitad de los años sesenta-, existe una gran heterogeneidad en cuanto a su extensión y a la duración de cada una de sus distintas fases (véase el gráfico 2). En general, se estima que en los países más avanzados en el proceso de transición demográfica el periodo del bono será menos extenso que en aquellos que se encuentran más atrasados. En Cuba y Chile, por ejemplo, se espera que el bono termine antes del primer cuarto del siglo; en Brasil y Colombia se va a extender hasta cerca de 2040, mientras que en El Salvador y Nicaragua está previsto que termine alrededor de 2050. En cinco países, entre los cuales se destacan el Estado Plurinacional de Bolivia y Guatemala, la duración del bono se extenderá más allá de la mitad de este siglo -en el primero se espera que el bono termine en el año 2060, mientras que en el segundo en 2067-. Uruguay, por otro lado, representa un caso especial, ya que en este país no se vislumbra un período de bono demográfico. Mientras que para el promedio de América Latina la relación de dependencia bajó un 38\% entre su valor máximo y su valor mínimo, en Uruguay el descenso fue solamente del 9\%. Además, después de alcanzar un valor máximo significativamente menor que el promedio regional, la relación de dependencia en este país se mantuvo siempre por encima de 2 personas dependientes para cada 3 en edades activas (véase el cuadro 2). El descenso relativamente suave de los niveles de fecundidad y la fuerte emigración de población en edades activas son factores relacionados con esta situación.

Actualmente, la población de América Latina se encuentra, en promedio, en la segunda y más favorable fase del bono demográfico, en la que la relación de dependencia alcanza niveles relativamente bajos y se mantiene descendente. Esta fase se inició alrededor de principios del siglo XXI y se espera que dure hasta finales de la próxima década, cuando comience la tercera, cuya duración está prevista hasta comienzos de los años 2040. Sólo en dos países, Cuba desde principios de los años noventa y Brasil a partir de 2007, el bono demográfico ya se adentró en la tercera etapa. En Chile se prevé que el inicio de esta fase se dará muy pronto, a partir de 2011. Además de Chile, otros seis países se encuentran en la segunda fase del bono -Colombia, Costa Rica, México, República Bolivariana de Venezuela, Panamá y Perú-, mientras los restantes siguen todavía en la primera. Es interesante notar que entre estos últimos se encuentran casos bastante disímiles, como los de Argentina y Guatemala. Mientras en Argentina la estructura etaria es relativamente envejecida, como resultado de una transición de la fecundidad que ya es bastante antigua, pero que ha sido relativamente suave, en Guatemala la estructura etaria es mucho más joven y el proceso de reducción de la fecundidad mucho más reciente.

\section{Impacto económico del bono demográfico en los sectores sociales}

Mientras que en la sesión anterior se analizó el bono demográfico a través de la tasa de dependencia demográfica, en la presente se hace desde la perspectiva de las tasas de dependencia económica (cociente entre consumidores y productores), empleando información sobre los patrones por edad del consumo y la producción. Gracias a estas relaciones es posible cuantificar el impacto del cambio demográfico y establecer una comparación con el ingreso y el consumo, el crecimiento del PIB y las tasas fiscales. El análisis realizado se basa en datos de las cuentas nacionales de transferencias disponibles para tres economías latinoamericanas -Chile, Costa Rica y Uruguay-. Las cuentas nacionales de transferencias representan un sistema contable compatible con las cuentas nacionales, que permite hacer estimaciones sobre los flujos económicos entre diferentes grupos de edades. Para ello se utilizan datos sobre ingresos, gastos y transferencias públicas y privadas, considerando las distintas instituciones que median en estas transacciones, 
o sea, el Estado, el mercado y la familia (MASON y otros, 2008). ${ }^{3}$ A partir de estos datos se definió un patrón de edad generalizado de la actividad económica, y se estimó el impacto de los cambios demográficos en los países de la región, variándose la estructura etaria de la población y manteniéndose constante el patrón de actividad económica. Si bien los niveles de gasto y consumo varían de un país a otro, debido a sus diferentes características económicas, el patrón general por edad suele ser bastante similar entre ellos, lo que justifica la utilización de los promedios de estos tres países latinoamericanos para proyectar los efectos económicos de los cambios en la estructura por edades de la población en los demás países de la región.

En las etapas iniciales de la transición demográfica, a medida que disminuye la fecundidad, se reduce el número de niños respecto al de los adultos en edad de trabajar, lo que, en términos económicos, significa un número decreciente de consumidores respecto del número de productores. En igualdad de condiciones, esta situación provoca un aumento de los ingresos laborales en relación con el consumo. El "bono demográfico" se ha asociado a esta liberación de recursos, y a este período, que puede durar varias décadas, se le ha denominado "ventana demográfica de oportunidades".

El período de bono demográfico termina cuando empieza a aumentar de manera pronunciada el número de personas mayores respecto al de los adultos en edad de trabajar. En este caso, el número de consumidores crece respecto al número de productores, y si no se compensa la disminución de ingresos laborales por consumidor con ingresos no provenientes del trabajo (como el aumento del ahorro), podría producirse un descenso del bienestar económico, y el período de bono demográfico daría paso a otro de "desventaja demográfica".

No obstante, el impacto económico a largo plazo de estos cambios demográficos dependerá de las medidas de política que tomen los gobiernos y los cambios de comportamiento de las personas. El bono demográfico puede consumirse totalmente, provocando un aumento temporal del consumo per cápita o, alternativamente, puede ser invertido en capital humano y físico, generando un aumento permanente del consumo per cápita a largo plazo. En este último caso, se estaría aprovechando el bono demográfico para prepararse para el surgimiento de sociedades más envejecidas.

\section{Dependencia económica en las etapas iniciales y finales de la vida}

Los potenciales beneficios económicos del bono demográfico y los posibles aspectos negativos del envejecimiento de la población resultan del ciclo de vida económica -es decir, del patrón de actividad económica por edades-, que en general es muy similar entre los países, pero con algunas variaciones significativas. En el gráfico 3 se muestra este ciclo en base a la información de Chile, Costa Rica y Uruguay. La actividad económica se mide respecto al promedio de los ingresos laborales entre los 30 y los 49 años en cada una de las economías, para uniformizar la medida en todos los países. La línea discontinua representa el promedio de los ingresos laborales por edades. Estos se refieren a los ingresos medios por persona, no por trabajador, y el promedio se calcula incluyendo a todas las personas de una determinada edad. Por lo tanto, los perfiles no sólo reflejan los efectos de las tasas de participación laboral, sino también de las tasas de desempleo. Los ingresos laborales equivalen a cero en el caso de los niños, registran un aumento pronunciado alrededor de los 20 años, debido al ingreso en el mercado laboral y al término de la escolaridad formal, y permanecen relativamente estables en el grupo de los 35 a los 50 años.

Esta tendencia es muy distinta de la de Estados Unidos y los países europeos (LEE; LEE; MASON, 2007), cuyos perfiles suelen mostrar un aumento de los ingresos labo-

\footnotetext{
3 El desarrollo de las cuentas nacionales de transferencias de Chile, Costa Rica y Uruguay se enmarca en el proyecto Transferencias intergeneracionales, envejecimiento de la población y protección social en América Latina, coordinado por el CELADE (véase nota de pie n. 1).
} 
GRÁFICO 3

Ciclo de vida económica promedio de 3 países de América Latina (Chile, Costa Rica y Uruguay)

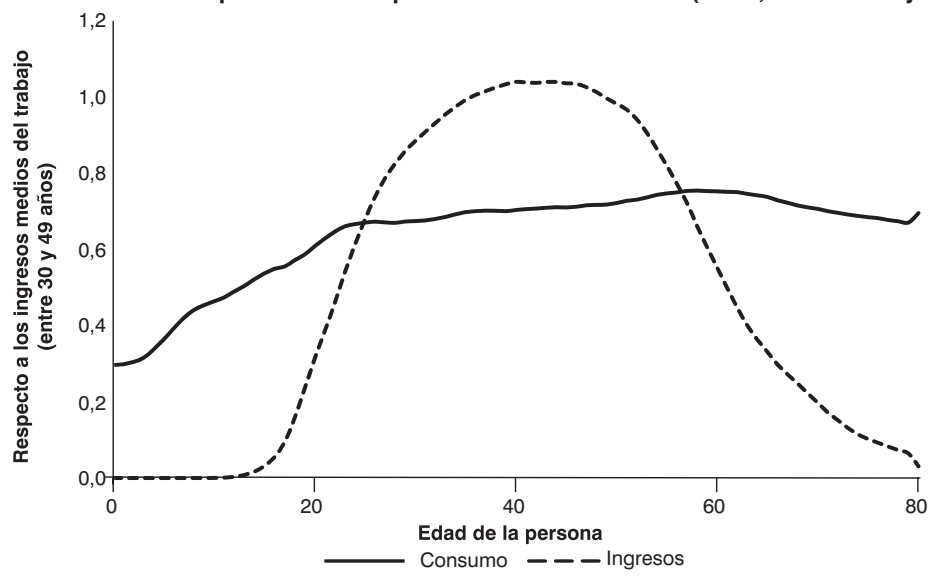

Fuente: Centro Latinoamericano y Caribeño de Demografía (CELADE) - División de Población de la CEPAL, Estimaciones y proyecciones de población, 2007 e información económica del proyecto conjunto ECLAC-IDRC sobre transferencias intergeneracionales, envejecimiento de la población y protección social en América Latina.

rales con la edad, lo que refleja en parte la retribución de la experiencia y la educación. Los ingresos empiezan a disminuir a una edad más avanzada, como consecuencia de la jubilación y la salida del mercado laboral. En los países con niveles más altos de cobertura de pensiones se observa una disminución mucho más acentuada de la actividad laboral, centrada en edades obligatorias de jubilación. En las economías de América Latina, en cambio, las personas mayores suelen seguir dependiendo de los ingresos laborales, y muchas trabajan mientras su estado físico se lo permite.

La línea continua del gráfico indica los niveles de consumo medio por edad. Un importante componente de esta medida se refiere al consumo de bienes y servicios públicos, sobre todo la atención de la salud y la educación pública. Los niveles de consumo son bajos en el caso de los niños y aumentan de forma casi lineal durante la infancia. Los efectos de los gastos de educación de los niños pueden apreciarse en el incremento más rápido del consumo en torno a los 5 y 6 años, a medida que comienzan la educación preescolar y la escuela primaria. El consumo aumenta lentamente durante la etapa adulta y alcanza su punto máximo antes de llegar a los 60 años, para disminuir posteriormente en edades más avanzadas, con un notable aumento en las personas muy mayores (80 años y más), debido principalmente al incremento de los gastos de salud en este grupo etario. Este patrón de consumo entre las personas mayores contrasta marcadamente con el observado en Estados Unidos y Japón, donde aumenta de manera muy acentuada con la edad, reflejando un mayor consumo en salud pública y privada en esos países (LEE; LEE; MASON, 2007).

Como se aprecia en el gráfico 3 , existen tres períodos distintos de vida económica. Los niños y los adultos jóvenes, antes de formar parte de la fuerza laboral, dependen de las transferencias de los padres o del gobierno para cubrir sus necesidades de consumo. Este período de dependencia económica dura alrededor de 25 años, y alcanza su punto máximo (mayor distancia entre las líneas de consumo y de ingreso laboral) a los 15 años. La ayuda a estos adolescentes equivale a casi la mitad de los ingresos anuales del trabajo de los adultos de mediana edad. ${ }^{4}$ Tras este máximo, la de-

\footnotetext{
${ }^{4}$ Es importante considerar que las cuentas de transferencias nacionales, al igual que las cuentas nacionales, carecen de información sobre el empleo del tiempo. Se cree que la incorporación de datos sobre esa variable aumentaría el nivel de dependencia de los infantes respecto al de los adolescentes.
} 
pendencia económica desciende de manera constante con el término de la escolaridad y el ingreso a la fuerza laboral. Alrededor de los 25 años la mayoría de las personas goza de independencia económica. En el otro extremo, las personas mayores, que en su mayoría han dejado de formar parte de la fuerza laboral, dependen de las transferencias privadas o públicas para atender sus necesidades de consumo, aunque parte de ellas podría también financiarlas durante los años de la jubilación a través de los ahorros acumulados. El período de dependencia económica de las personas mayores se inicia poco antes de llegar a los 60 años y aumenta de forma constante, para alcanzar un nivel máximo en el último intervalo de edad, a partir de los 80 años. En esta edad avanzada, las personas alcanzan un nivel máximo de dependencia económica, que exige una ayuda equivalente a dos tercios de los ingresos laborales anuales de los adultos de mediana edad. Entre estos dos períodos de dependencia económica existe otro de independencia de unos 30 años, que abarca desde los 25 a los 55 años aproximadamente, en el que los ingresos laborales superan al consumo.

La dependencia económica al principio $y$ al final del ciclo de vida se torna factible gracias al período de 30 años de excedente de producción durante las edades más productivas. Son tres los mecanismos principales mediante los cuales se reasignan los recursos: las transferencias familiares, las gubernamentales y las intertemporales, a través de los mercados financieros, en forma de ahorro y préstamos. Las transferencias familiares pueden realizarse dentro de un mismo hogar, por ejemplo, cuando los padres proporcionan alimento, vestido y cobijo a sus hijos. También pueden efectuarse entre hogares, como cuando un trabajador migrante envía remesas a su familia o, tras el fallecimiento de los padres, a través de una herencia a los hijos adultos.

Las transferencias gubernamentales suponen el pago de impuestos por parte de un grupo de la población (generalmente los trabajadores) para sufragar los recursos ofrecidos a otras personas. Estas transferencias de recursos pueden adoptar la forma de pagos en efectivo, como pensiones, presta- ciones sociales y de desempleo, o de beneficios en especie, como educación pública, hospitales públicos y formación laboral. Si bien los impuestos se aplican principalmente a la población en edad de trabajar, las prestaciones estatales se dirigen sobre todo a los jóvenes y las personas mayores. El último mecanismo de transferencia se da a través de la utilización de los mercados financieros para el ahorro y el préstamo.

Las transferencias, tanto privadas como públicas, funcionan por lo general como sistemas de reparto, en los que el monto total de las transferencias de beneficios recibidas por las personas es igual al monto total de las transferencias realizadas. Manteniéndose constantes otros factores, los cambios en la estructura por edades modifican el número relativo de receptores y donantes en la sociedad. Esta variación en la proporción de receptores y donantes genera el bono demográfico.

\section{La magnitud económica del bono demográfico}

La magnitud económica del bono demográfico se estima a través del efecto de la variación de la estructura por edades en el cociente entre consumidores y productores. Este cociente se conoce como tasa de dependencia económica, y mide la relación entre el número efectivo de consumidores y el número efectivo de productores. El número efectivo de consumidores se calcula ponderando la distribución etaria de la población de cada país por el perfil de edad estándar de consumo, basado en el promedio simple de Chile, Costa Rica y Uruguay. De igual manera, el número efectivo de productores se calcula utilizando el perfil de edad estándar de los ingresos laborales. De esta forma, el análisis se centra exclusivamente en el impacto económico surgido a partir de las diferencias demográficas entre los países.

La tasa de dependencia económica puede expresarse como:

$\mathrm{C}(\mathrm{t}, \mathrm{i}) / \mathrm{P}(\mathrm{t}, \mathrm{i})=\Sigma\{\mathrm{c}(\mathrm{x}) * \mathrm{n}(\mathrm{x}, \mathrm{t}, \mathrm{i})\} / \Sigma\{1(\mathrm{x}) * \mathrm{n}(\mathrm{x}, \mathrm{t}, \mathrm{i})\}$ Donde $\mathrm{C}(\mathrm{t}, \mathrm{i})=$ Número efectivo de consumidores en el año t y el país $\mathrm{i}$; $\mathrm{P}(\mathrm{t}, \mathrm{i})=$ Número efectivo de productores en el año $t$ y el país $\mathrm{i} ; \mathrm{c}(\mathrm{x})=$ Consumo medio en la edad 
$\mathrm{x}$ del perfil estándar; $1(\mathrm{x})=$ Ingresos medios del trabajo en la edad $x$ del perfil estándar, y $n(x, t, i)=$ Población total de edad $x$, en el año t y el país i.

Si se presupone que las necesidades de consumo de los niños y las personas mayores se financian mediante transferencias de la población en edad de trabajar, la disminución de esta tasa de dependencia económica representa una liberación de recursos para su uso en consumo e inversiones. En el gráfico 4 se observa la tendencia general de la tasa de dependencia económica de la región en su conjunto. El cociente entre consumidores y productores aumentó entre 1950 y 1971, lo que refleja una proporción creciente de niños (consumidores netos) en la población. Aquel año alcanzó un máximo de aproximadamente 1,5 consumidores por productor, y desde entonces ha disminuido de manera constante, revelando el decrecimiento de la proporción de niños. Basándose en las proyecciones de población del Celade, que prevén una disminución constante de la fecundidad, se espera que la tasa de dependencia económica alcance su punto más bajo en 2025, con aproximadamente 1,2 consumidores por productor, una disminución de alrededor del $20 \%$ respecto de 1971 . Este valor representa el volumen del bono demográfico: un aumento del $20 \%$ de los ingresos laborales por consumidor durante un período de 54 años, o el equivalente a un crecimiento medio anual de aproximadamente el 0,4\% del consumo por persona. Se prevé que a partir de 2025 el cociente entre consumidores y productores aumente, como consecuencia del notable incremento de la proporción de personas mayores en la región, provocando una disminución del consumo por persona.

Esta tendencia regional esconde profundas diferencias entre un país y otro. En el gráfico 5 se resumen cuatro tendencias distintas de la tasa de dependencia económica en América Latina, definidas a partir de la aplicación del algoritmo k-medias ${ }^{5}$ de Hartigan y Wong (1979), utilizándose el software estadístico R (The R Project for Statistical Computing). La línea discontinua representa la tendencia general para América Latina y el Caribe. La mayoría de los países se ajusta a la tendencia I, que es similar a la general, con la salvedad que en ellos la tasa de dependencia económica entre

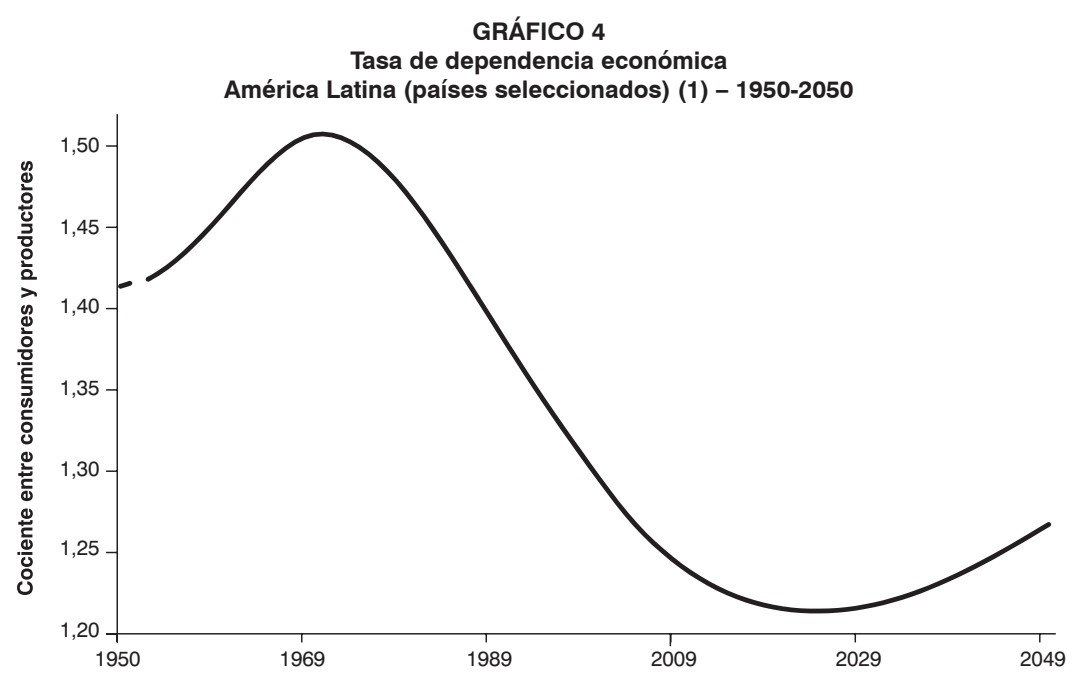

Fuente: Centro Latinoamericano y Caribeño de Demografía (Celade) - División de Población de la Cepal, Estimaciones y proyecciones de población, 2007 e información económica del proyecto conjunto ECLAC-IDRC sobre transferencias intergeneracionales, envejecimiento de la población y protección social en América Latina.

(1) Incluye algunos países del Caribe.

\footnotetext{
${ }^{5} \mathrm{El}$ algoritmo k-medias tiene por objetivo dividir un conjunto de puntos en $\mathrm{k}$ grupos, de manera tal a minimizar la suma de los cuadrados de las distancias entre cada punto y el valor promedio del grupo.
} 
1960 y 1990 fue algo superior, debido a su estructura inicial por edades más joven. En la tendencia II, que corresponde al Estado Plurinacional de Bolivia, Guatemala, Haití, Honduras, Nicaragua y Paraguay, se observa la misma trayectoria que en la tendencia regional, pero con una demora de una o dos décadas, como consecuencia de su retraso en la transición demográfica. Los países de la tendencia III (Cuba y Chile) registran una disminución mucho más rápida de la tasa de dependencia económica y alcanzan antes un nivel mínimo, a causa del rápido envejecimiento de su población. En todos los países se producirá un aumento de las tasas de dependencia económica a medida que la proporción de personas mayores se incremente. En Cuba y Chile, que se encuentran bien avanzados en la transición demográfica, ya se observan aumentos pronunciados de estas tasas en la actualidad. La tendencia IV representa a la Argentina y
Uruguay. En ella se observa muy poca variación en la tasa de dependencia económica a lo largo del tiempo, puesto que en esos países la población era relativamente más envejecida al inicio del período.

Los gráficos 4 y 5 muestran un siglo de cambios en la tasa de dependencia económica en América Latina. Sin embargo, resulta útil también considerar la situación en que se encuentran actualmente los diferentes países de la región en este proceso de transformación. Para ello se mide la magnitud del bono demográfico observado en la última década y previsto para la próxima en cada país (véase el gráfico 6). En el pasado decenio, el cambio demográfico contribuyó positivamente al crecimiento del bienestar económico en casi todos los países de América Latina. El bono demográfico para la región en su conjunto fue equivalente a un aumento de aproximadamente el $6 \%$ de los ingresos la-

GRÁFICO 5

Cuatro tendencias típicas de la tasa de dependencia económica América Latina - 1950-2050
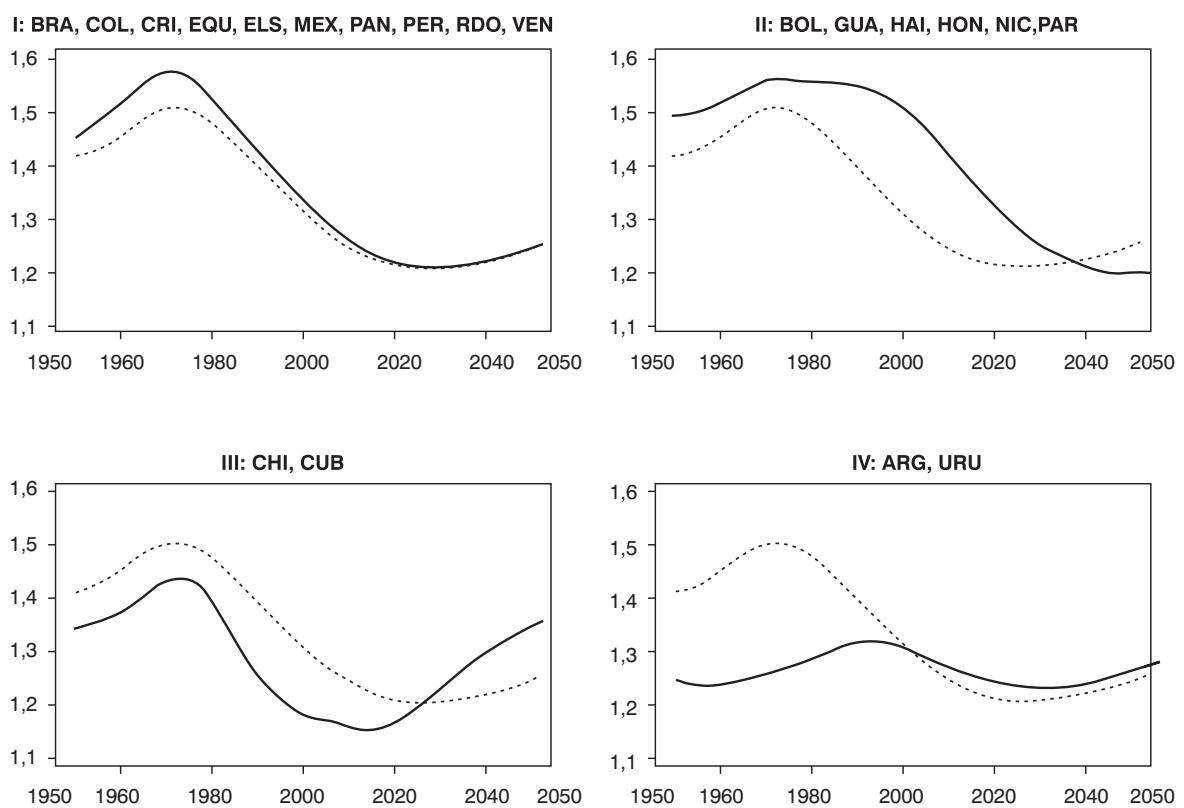

Fuente: Centro Latinoamericano y Caribeño de Demografía (CELADE) - División de Población de la CEPAL, Estimaciones y proyecciones de población, 2007 e información económica del proyecto conjunto ECLAC-IDRC sobre transferencias intergeneracionales, envejecimiento de la población y protección social en América Latina.

Nota: La línea discontinua se refiere a la tasa promedio de América Latina 
borales por consumidor entre 1998 y 2008 , lo que representó más de una tercera parte del volumen de crecimiento general del PIB per cápita en ese período, estimado en aproximadamente el 16\% (Cepal, 2008a). Los países con mayor bono demográfico en ese lapso fueron Nicaragua $(+10 \%)$,
El Salvador $(+8,6 \%)$ y México $(+8,6 \%)$, mientras que los que registraron menor bono demográfico fueron Cuba $(+1,3 \%)$, Uruguay $(+1,6 \%)$ y Chile $(+2,5 \%)$.

En la década posterior a 2008 se espera un bono demográfico notablemente inferior para la región en su conjunto, con

\section{GRÁFICO 6}

Bono demográfico (aumento porcentual estimado de los ingresos laborales por consumidor debido a cambios demográficos)

Países de América Latina - 1998-2018
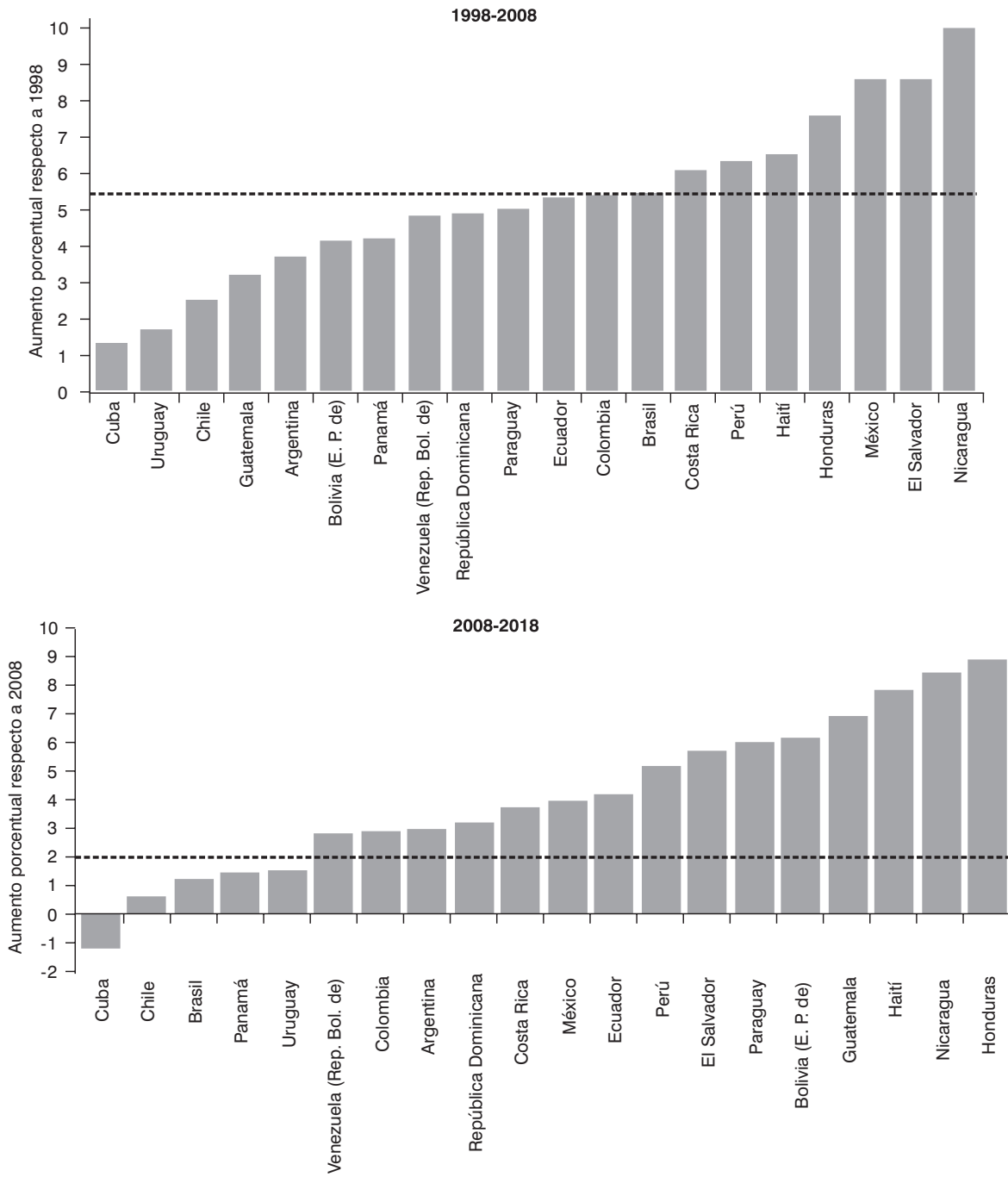

Fuente: Centro Latinoamericano y Caribeño de Demografía (CELADE) - División de Población de la CEPAL, Estimaciones y proyecciones de población, 2007 e información económica del proyecto conjunto ECLAC-IDRC sobre transferencias intergeneracionales, envejecimiento de la población y protección social en América Latina.

Nota: El promedio regional (línea discontinua) incluye países del Caribe. 
un aumento de los ingresos laborales por consumidor equivalente a menos del 3\% durante todo ese lapso. No obstante, existe una considerable heterogeneidad entre los países. Mientras en algunos como Honduras, Nicaragua y Haití se prevén bonos todavía significativos, de alrededor del $8 \%$ al $9 \%$, en otros el período de bono demográfico estará llegando a su fin, o incluso habrá una disminución de los ingresos laborales por consumidor, como en el caso de Cuba.

Este análisis muestra diferencias considerables en la magnitud del bono demográfico entre los países, que reflejan sus distintas etapas de transición demográfica. Sobre la base de este examen, se puede afirmar que el bono demográfico ha contribuido positivamente al bienestar económico general de la región durante la última década. Las previsiones para el próximo decenio son buenas en la mayoría de los casos, pero algunos países ya han entrado en una etapa de envejecimiento de su población en la que el cambio demográfico reduce las posibilidades de consumo, al aumentar la presión fiscal sobre las transferencias $/ m$ pacto económico del bono demográfico en tres sectores clave

Prácticamente todos los países de la región se enfrentan con problemas para ampliar la cobertura de sus sistemas de educación, salud y pensiones. En la presente sección se analiza en qué medida los cambios demográficos contribuyen a disminuir o aumentar esos retos. Para medir el impacto del bono demográfico en cada uno de esos sistemas de transferencia, se utilizan tasas de dependencia similares a las empleadas en el método considerado en la sección anterior. Se presupone que cada uno de los sectores se financia mediante sistemas de transferencia de reparto (con independencia de que las contribuciones procedan directamente de las familias o de los impuestos), y la tasa de dependencia del sector se define de forma análoga a la tasa de dependencia económica. Al igual que en ese cociente, el denominador se refiere al número efectivo de productores en la población (que proporcionan los recursos para las transferencias), pero el numerador ahora se refiere al número efectivo de beneficiarios del sector considerado (educación, salud o pensiones). Los beneficiarios efectivos se miden utilizando la información sobre el gasto medio en educación, atención de la salud y prestaciones de pensiones por edades, procedente de las cuentas de transferencias nacionales de Chile, Costa Rica y Uruguay.

Por lo tanto, la tasa de dependencia de cada uno de los sectores está definida por: $\mathrm{B}(\mathrm{t}, \mathrm{i}) / \mathrm{P}(\mathrm{t}, \mathrm{i})=\Sigma\{\mathrm{b}(\mathrm{x}) * \mathrm{n}(\mathrm{x}, \mathrm{t}, \mathrm{i})\} / \Sigma\{1(\mathrm{x}) * \mathrm{n}(\mathrm{x}, \mathrm{t}, \mathrm{i})\}$ Donde

$\mathrm{B}(\mathrm{t}, \mathrm{i})=$ Número efectivo de beneficiarios del sector en el año t y el país i;

$\mathrm{P}(\mathrm{t}, \mathrm{i})=$ Número efectivo de productores en el año t y el país i;

$\mathrm{b}(\mathrm{x})=$ Beneficios medios del sector en la edad $\mathrm{x}$ del perfil estándar;

$1(\mathrm{x})=$ Ingresos medios del trabajo en la edad $\mathrm{x}$ del perfil estándar, y

$\mathrm{n}(\mathrm{x}, \mathrm{t}, \mathrm{i})=$ Población total de edad $\mathrm{x}$, en el año t y el país i.

La tasa de dependencia del sector representa el porcentaje de ingresos laborales que se transfieren (o los impuestos que han de aplicarse) para financiar el respectivo programa de beneficios (educación, salud o pensiones), con los niveles actuales de cobertura y prestaciones. La disminución de la tasa de dependencia del sector supone una liberación de recursos. Este bono puede utilizarse dentro del mismo sector, para aumentar los beneficios (mediante la ampliación de la cobertura o las prestaciones por beneficiario) o reducir la carga impositiva. También podría emplearse en otros sectores.

En el gráfico 7 se muestra el promedio de servicios de educación, atención de la salud y pensiones recibidas por edades en tres economías de América Latina (Chile, Costa Rica y Uruguay). Aunque los niveles de estos programas varían de un país a otro en función de las distintas coberturas y la generosidad de los programas sociales, el patrón general por edad suele ser bastante similar entre ellos. Esto justifica la utilización de los promedios de las tres economías de América Latina para proyectar los efectos de los cambios en la estructura por edades de la población en la educación, la salud y las pensiones de los demás países de la región. Se presupone que esos programas se finan- 


\section{GRÁFICO 7}

Consumo promedio por edad en educación, atención de salud y pensiones de 3 países de América Latina (Chile, Costa Rica y Uruguay)

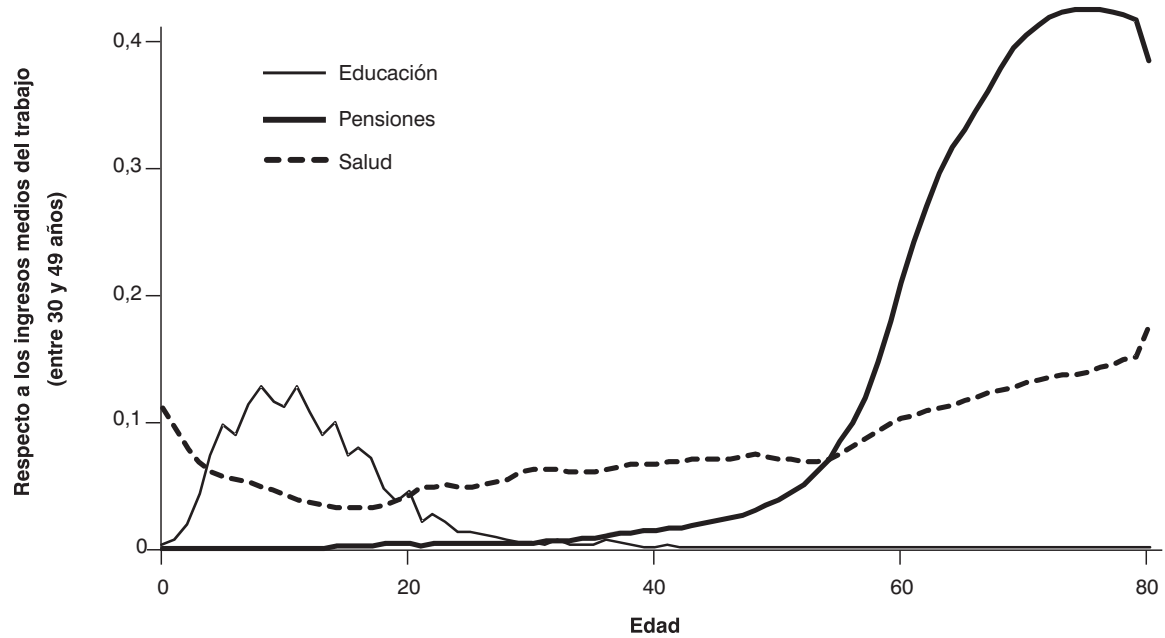

Fuente: Centro Latinoamericano y Caribeño de Demografía (CELADE) -División de Población de la CEPAL, Estimaciones y proyecciones de población, 2007 e información económica del proyecto conjunto ECLAC-IDRC sobre transferencias intergeneracionales, envejecimiento de la población y protección social en América Latina.

cian con los ingresos laborales (mediante contribuciones familiares o imposiciones estatales). En lo que respecta a los grupos de edades, el consumo en educación se concentra en los jóvenes de entre 5 y 20 años; las prestaciones de jubilación se dirigen a las personas mayores y la atención de la salud muestra por lo general la forma de una $\mathrm{U}$, con un gran consumo en los infantes, un menor consumo en la adolescencia y uno mayor entre las personas de edad.

\section{Impacto económico del bono demográfico en el sector de educación}

Para analizar el impacto de los cambios demográficos en las transferencias del sector educativo se utiliza la tasa de dependencia de la educación, es decir, el cociente entre el número efectivo de estudiantes y el número efectivo de productores. El número efectivo de estudiantes se calcula aplicando el perfil de edad estándar del gasto en educación (basándose en los datos de Chile, Costa Rica y Uruguay) a la distribución por edades de la población de cada país. El número de productores, en tanto, se calcula de manera similar, utilizando el perfil de edad estándar de los ingresos laborales.
La tasa de dependencia de la educación en la región en su conjunto se situó en el $10 \%$ en 1950 , lo que significa que por entonces se necesitaba un impuesto del 10\% sobre los ingresos laborales para ayudar a los estudiantes, con los actuales niveles de matrícula. En los años cincuenta y sesenta la tasa se incrementó, debido al aumento en el volumen de la población en edad escolar respecto de aquella en edad de trabajar. En 1971 alcanzó un nivel máximo del 11,7\%. Posteriormente, se inició una retracción a largo plazo, como consecuencia de la transición de la fecundidad en la región. Actualmente se sitúa en el $6,8 \%$, lo que supone una disminución del $40 \%$ respecto del valor máximo. En otras palabras, el mismo nivel de escolarización por niño puede ahora lograrse utilizando un $40 \%$ menos de recursos. Se prevé que la fecundidad siga descendiendo en la región y lleve a una tasa de dependencia de la educación del 4,2\% para 2050, lo que representaría una disminución adicional del $40 \%$ respecto de los valores actuales.

En el gráfico 8 se comparan cuatro tendencias subregionales de América Latina (línea continua) con la tendencia regional (línea discontinua) durante el periodo 19502050. La mayoría de los países se ajusta a 
la tendencia I, que coincide con el patrón regional. Los países de la tendencia II (el Estado Plurinacional de Bolivia, El Salvador, Honduras, Nicaragua, Paraguay y la República Dominicana) muestran un comportamiento similar al de la región, aunque con valores notablemente superiores, debido a su fecundidad más alta. La tendencia III, que corresponde a Guatemala y Haití, registró valores sistemáticamente elevados de dependencia de la educación entre los años 1950 y 1990, a causa de sus altos niveles de fecundidad. En los países de la tendencia IV (Argentina, Chile, Cuba y Uruguay), la tasa de dependencia de la educación en los años cincuenta era mucho más baja que la de la región, porque su fecundidad ya era bastante inferior a la de los demás países.

Al analizar las tendencias nacionales durante la década pasada se observa un bono demográfico sustancialmente positivo en la educación en todos los países de la región, que refleja la disminución de la población en edad escolar respecto de aquella en edad de trabajar. El bono demográfico en educación fue particularmente amplio en México y Nicaragua, con aumentos superiores al $25 \%$. En toda la región, la disminución de la población en edad escolar respecto de la fuerza laboral supuso un bono en educación del $+19 \%$ durante la década pasada, lo que indica que la escolaridad pudo haber aumentado un $19 \%$ entre 1998 y 2008 sin un aumento de la carga financiera.

Las perspectivas futuras hasta 2018 prevén la manutención de amplios bonos demográficos en el sistema educativo, con un valor total del $+16 \%$ para la región en su conjunto. Durante este periodo se esperan bonos en educación especialmente amplios en Nicaragua y Honduras, con valores de alrededor del $+25 \%$.

\section{Impacto económico del bono demográfico en el sector de salud}

El gasto en la atención de la salud suele concentrarse en los niños y las personas

\section{GRÁFICO 8}

Cuatro tendencias típicas de la tasa de dependencia de la educación América Latina - 1950-2050

I: BRA, COL, CRI, ECU, MEX, PAN, PER, VEN

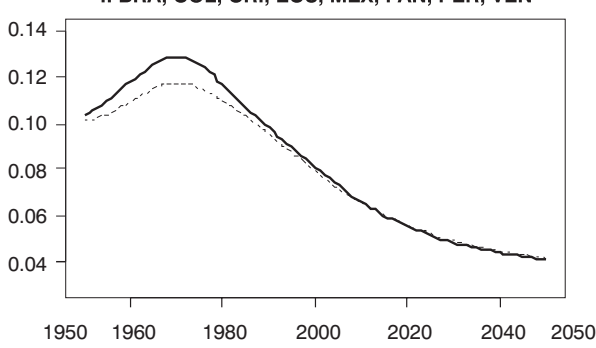

III: GUA, HAI

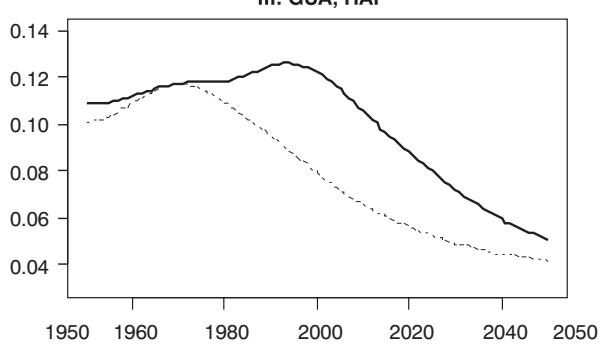

II: BOL, ELS, HON, NIC, PAR, RDO

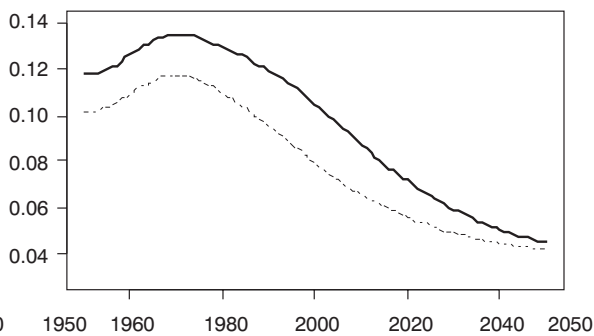

IV: ARG, CHI, CUB, URU

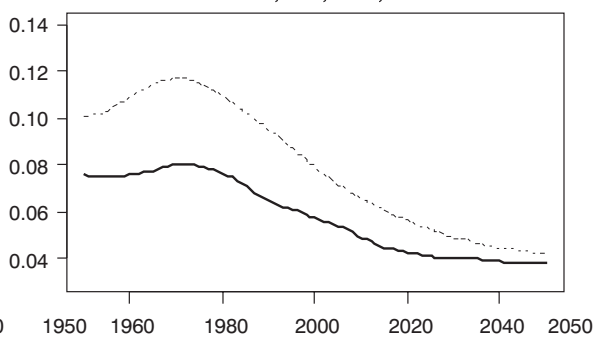

Fuente: Centro Latinoamericano y Caribeño de Demografía (Celade) - División de Población de la Cepal, Estimaciones y proyecciones de población, 2007 e información económica del proyecto conjunto ECLAC-IDRC sobre transferencias intergeneracionales, envejecimiento de la población y protección social en América Latina.

Nota: La línea discontinua se refiere a la tasa promedio de América Latina. 
mayores. Cuando baja la fecundidad y la proporción de niños disminuye, las fuerzas demográficas contribuyen a reducir sustancialmente la presión financiera sobre los sistemas de salud durante un período de varias décadas; en cambio, cuando la población empieza a envejecer y la proporción de personas mayores se incrementa de forma rápida y sostenida, estas fuerzas aumentan bruscamente la presión fiscal sobre este sistema. Por ejemplo, según estimaciones de las cuentas de transferencias nacionales, el consumo en salud promedio de una persona mayor en América Latina es el doble de la de un adulto en edad de trabajar. Mientras en 1970 el gasto en salud de las personas mayores en la región en su conjunto habría representado una décima parte, aproximadamente, de todo el gasto en este servicio, en 2030 se prevé que habrá aumentado a una cuarta parte (Cepal, 2008a).

La tasa de dependencia de la atención de la salud mide la relación entre el número de consumidores de este servicio y el número de productores totales en la economía. En 1950, para la región en su conjunto, esta tasa se situó en el 15,6\%, lo que significa que para proporcionar el nivel de atención de la salud registrado actualmente en Chile, Costa Rica y Uruguay habría sido necesario aplicar un impuesto del $15,6 \%$ a los ingresos laborales. La tasa aumentó ligeramente durante los años cincuenta y sesenta, al incrementarse la proporción de niños, alcanzando un máximo del $16,8 \%$ en 1967 , antes de iniciar un largo período de declive, correspondiente al aumento de la proporción de personas en edad de trabajar. Actualmente se sitúa en el $13,3 \%$, y se prevé que alcance su nivel más bajo hacia 2015. A partir de ese momento, se proyecta un aumento que la llevará al 15,2\% en 2050.

Como se ha observado anteriormente, esta tendencia regional esconde una gran heterogeneidad demográfica entre los países. En el gráfico 9 se comparan cuatro tendencias subregionales (línea continua) con la tendencia regional (línea discontinua) de la tasa de dependencia de la atención de la salud durante el periodo 1950-2050. La mayoría de los países se ajusta a la tendencia I, similar a la regional, pero con tasas de dependencia más elevadas en los años cincuenta y sesenta, debido a la estructura por edades más joven de esas poblaciones. Los países de la tendencia II (el Estado Plurinacional de Bolivia, El Salvador, Guatemala, Haití, Honduras, Nicaragua y Paraguay) siguen un patrón similar al de la región, pero con un retraso de unas dos décadas por su transición demográfica menos avanzada. En Chile y Cuba, países que representan la tendencia III, la tasa bajo análisis sigue un comportamiento similar al regional, pero con niveles inferiores de 1950 a 2000, y a partir de entonces empieza a ascender bruscamente, debido al rápido envejecimiento de su población. Argentina y Uruguay, países del grupo IV, revelan una tendencia creciente de las tasas de dependencia de la salud, con niveles relativamente bajos al inicio del período y relativamente elevados al final en relación al promedio regional.

Durante la última década se registraron bonos positivos importantes en el sector sanitario de la mayoría de los países de América Latina. Sin embargo, en su conjunto, el bono de la salud en la región fue modesto $(+5 \%)$, y equivalió a una cuarta parte del observado en la educación. Para el próximo decenio se prevén diferencias considerables en relación a los efectos de los cambios en la estructura por edades en el financiamiento de la atención de la salud. En Guatemala, Haití y Honduras la disminución del número de niños como proporción de la población reducirá considerablemente la carga sobre este tipo de atención, con un bono del $+10 \%$ durante los próximos 10 años. En cambio, en países como Brasil, Chile y Cuba se prevé un aumento de la tasa de dependencia de la salud. Si no se realiza un mayor esfuerzo, los recursos por beneficiario durante la próxima década registrarán una contracción del 5\% en Cuba, $3 \%$ en Chile y $1 \%$ en Brasil, debido al envejecimiento de su población.

El cambio demográfico, además de afectar al financiamiento de la salud, repercutirá notablemente en el tipo de necesidades sanitarias de las personas. Esto significa que, además de enfrentarse a los nuevos problemas que suponen las enfermedades crónicas provocadas por el envejecimiento de la población, los países aún tendrán que 


\section{GRÁFICO 9}

Cuatro tendencias típicas de la tasa de dependencia de la atención de la salud América Latina - 1950-2050

I: BRA, COL, CRI, ECU, MEX, PAN, PER, RDO, VEN

II: BOL, ELS, GUA, HAI, HON, NIC, PAR
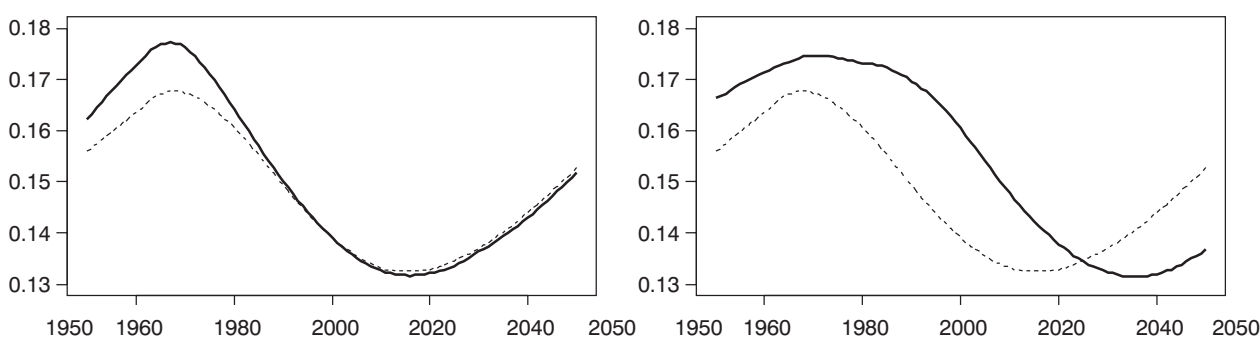

III: $\mathrm{CHI}$, CUB
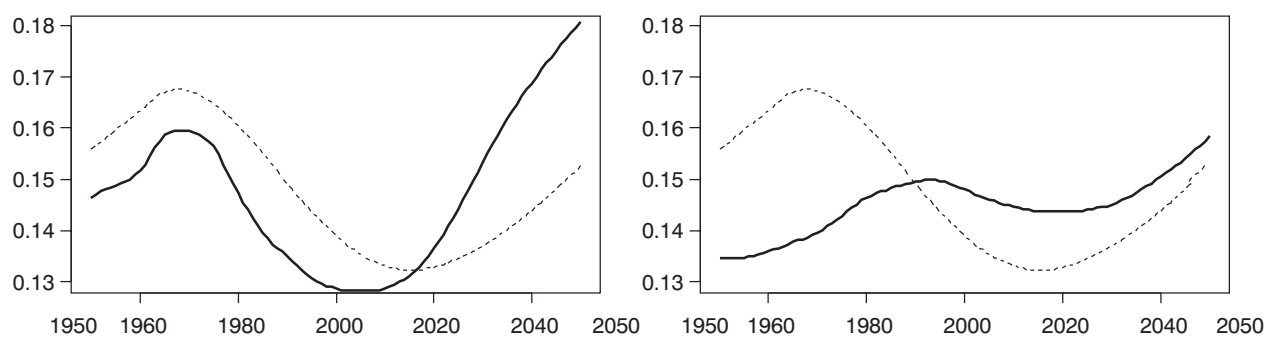

Fuente: Centro Latinoamericano y Caribeño de Demografía (Celade) - División de Población de la Cepal, Estimaciones y proyecciones de población, 2007 e información económica del proyecto conjunto ECLAC-IDRC sobre transferencias intergeneracionales, envejecimiento de la población y protección social en América Latina.

Nota: La línea discontinua se refiere a la tasa promedio de América Latina.

lidiar con la carga que suponen las enfermedades infecciosas típicas de las etapas tempranas de la transición epidemiológica. O sea, los sistemas de atención de la salud de la región tendrán que encarar la doble carga del "rezago epidemiológico" y el envejecimiento de la población, y serán objeto de una gran presión para ampliarse (Cepal, 2006).

\section{Impacto económico del bono demográfico en el sistema de pensiones}

Si bien los cambios demográficos provocarán una reducción sustancial de la carga fiscal asociada al financiamiento de la educación, y permitirán ampliar significativamente la cobertura en ese sector, estas mismas transformaciones también están dificultando de manera creciente el financiamiento de los sistemas de reparto de pensiones. En muchos países de la región, esta inquietud sobre la sostenibilidad fiscal a largo plazo de los sistemas públicos de pensiones condujo a su reforma, mediante la privatización total o parcial. En la presente sección se examinan los distintos efectos del cambio demográfico en estos sistemas de reparto, utilizando un perfil estándar de prestaciones de jubilación (basado en Chile, Costa Rica y Uruguay), y haciendo abstracción de las diferencias en las políticas previsionales de los países latinoamericanos.

La tasa de dependencia de las pensiones mide la relación entre el número de pensionados y el de productores. En la región en su conjunto, esta tasa se situó en el $7 \%$ en 1950 , lo que significa que aquel año habría sido necesario un impuesto del $7 \%$ de los ingresos laborales para apoyar a la población jubilada con los actuales niveles de cobertura y prestaciones. La presión demográfica sobre los sistemas de reparto de pensiones fue casi imperceptible durante la mayor parte del último siglo. La tasa de dependencia de las pensiones a nivel regional 
registró sólo un ligero aumento durante las últimas seis décadas, para situarse en el $9 \%$ en 2008. Recientemente, los efectos del envejecimiento de la población en América Latina han empezado a hacerse evidentes, y se prevé que esta tasa aumente bruscamente, situándose próxima al $20 \%$ en 2050 . Es decir, sin tener en cuenta el impacto adicional de la ampliación de la cobertura de las pensiones, el envejecimiento de la población haría que los impuestos destinados a las pensiones aumentaran más del doble, pasando del nivel actual del $9 \%$ de los ingresos laborales al $20 \%$ para mediados de siglo.

En el gráfico 10, que compara la tendencia regional (línea discontinua) de la tasa de dependencia de las pensiones con cuatro tendencias subregionales (línea continua) entre 1950 y 2050, se observa que la mayoría de los países de América Latina se ajusta a la tendencia I, que sigue de cerca la tendencia regional general. El Estado Plurinacional de Bolivia, Guatemala, Haití, Honduras y Para- guay, agrupados en la tendencia II, siguen un comportamiento similar al regional, pero con un retraso de dos o tres décadas a causa de su transición demográfica menos avanzada. Los países de la tendencia III (EI Salvador, Nicaragua, República Bolivariana de Venezuela y República Dominicana) también siguen la tendencia regional pero, debido a su estructura por edades ligeramente más joven, sus tasas de dependencia de las pensiones son inferiores a la media regional. Finalmente, los países que representan la tendencia IV (Argentina, Cuba y Uruguay) están varias décadas más avanzados que la tendencia regional.

Con la excepción de Haití, donde el cambio demográfico favoreció la expansión del sistema de pensiones (con un bono del $+3 \%$ ), todos los demás países de la región presentaron bonos negativos en los sistemas de pensiones durante la década pasada. Chile registró el valor más negativo de América Latina, con una pérdida del 15\%

GRÁFICO 10

Cuatro tendencias típicas de la tasa de dependencia de las pensiones América Latina - 1950-2050
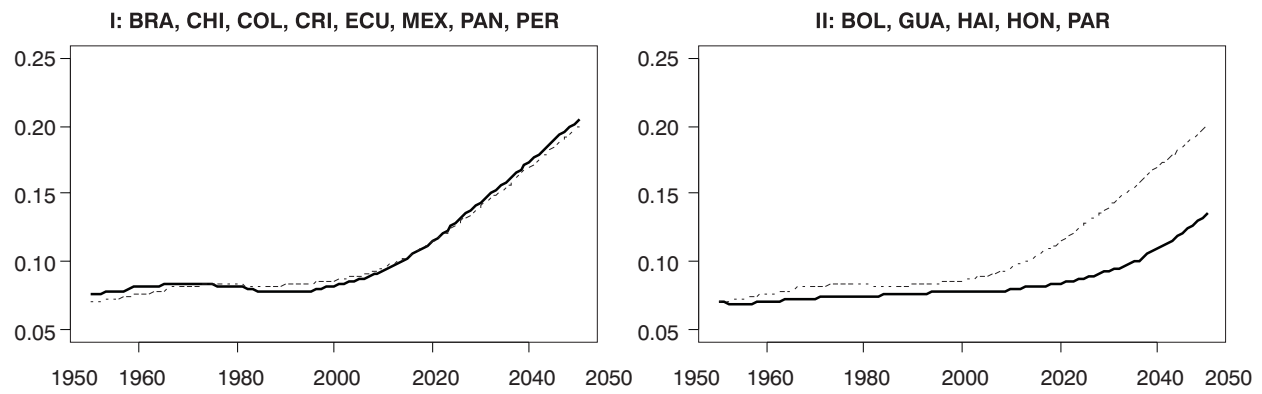

III: ELS, NIC, RDO, VEN
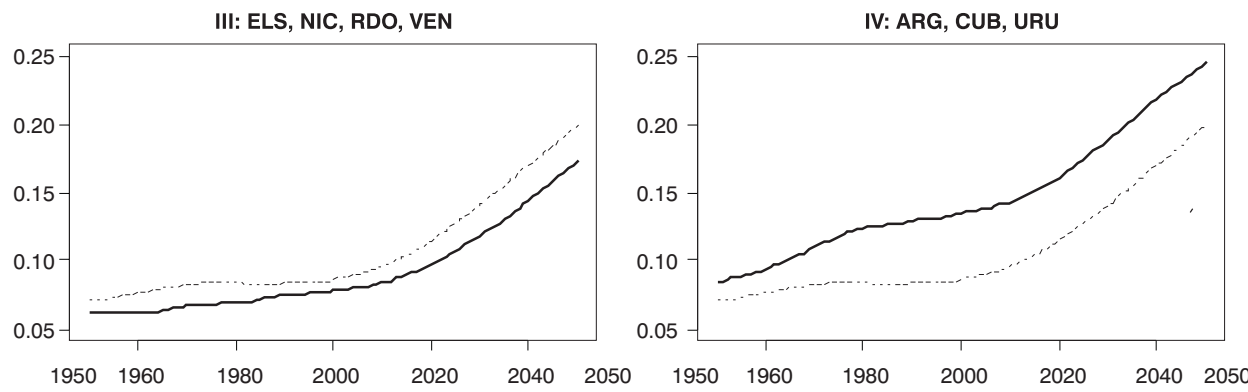

Fuente: Centro Latinoamericano y Caribeño de Demografía (Celade) - División de Población de la Cepal, Estimaciones y proyecciones de población, 2007 e información económica del proyecto conjunto ECLAC-IDRC sobre transferencias intergeneracionales, envejecimiento de la población y protección social en América Latina.

Nota: La línea discontinua se refiere a la tasa promedio de América Latina. 
durante el pasado decenio, seguido de la República Bolivariana de Venezuela (-13\%) y Cuba (-12\%). En esos países, la población jubilada aumentó mucho más rápido que aquella en edad de trabajar. En este mismo periodo, el bono en estos sistemas para la región en su conjunto fue del $-9 \%$, lo que implica que los impuestos destinados a financiar las pensiones tendrían que haber aumentado un $9 \%$ aproximadamente para apoyar a la creciente población de personas jubiladas con los actuales niveles de prestaciones y cobertura.

Se estima que el aumento de la tasa de dependencia de las pensiones dará lugar a un bono demográfico negativo cada vez más significativo en los sistemas de reparto de pensiones durante la próxima década. En este sentido sobresale el caso de Colombia, con pérdidas del $24 \%$, además de Brasil, Cuba, Chile, Costa Rica, México y la República Bolivariana de Venezuela, donde se prevén pérdidas de aproximadamente el $20 \%$ hasta el final del decenio que viene.

\section{Conclusiones}

América Latina se encuentra actualmente en medio a una coyuntura favorable para el desarrollo económico, determinada por el bono demográfico. En algunos países este período ya entró o está próximo a ingresar en una etapa terminal, mientras que en otros se encuentra en una fase todavía inicial, con perspectiva de extenderse por las próximas cinco o seis décadas. Así que, más tarde en algunos países y más temprano en otros, la ventaja de una relación favorable entre la población en edades activas y dependientes tenderá a modificarse, como resultado del incremento proporcional continuo de las personas mayores.

En términos económicos, si se parte del supuesto de que las necesidades de consumo de los niños y las personas mayores se solventan con transferencias provenientes de la población económicamente activa, el bono demográfico estaría determinado por la liberación de recursos económicos resultante de la caída de la relación de dependencia, que podría ser utilizada tanto en inversión como en consumo. Para la región en su conjunto, se estima que el bono demográfico entre 1971 y 2025 habrá sido de aproximadamente un $0,4 \%$ anual.

Sin embargo, el tamaño del bono demográfico depende del período analizado. En la década pasada (1998-2008), por ejemplo, el bono demográfico para la región en su conjunto fue de aproximadamente un 6\% de recursos económicos liberados por consumidor, lo que puede equipararse, para su mejor evaluación, a un tercio del aumento del PIB per cápita en el mismo período. Si bien este puede entenderse como un dividendo potencial relativamente modesto, no deja de ser un aporte significativo en un contexto de escaso crecimiento económico en la mayoría de los países de la región. Para la próxima década (2008-2018) se proyecta un bono demográfico sustancialmente más bajo, equivalente a menos del $3 \%$ de recursos económicos liberados por consumidor.

En suma podría decirse que, en general, el bono demográfico, aunque modesto, ha sido positivo para la región en la última década, y también lo será en la próxima, especialmente para aquellos países que aún tienen potencial para el descenso de la fecundidad y donde el envejecimiento es todavía incipiente. Para aquellos más envejecidos, en cambio, el bono demográfico habrá de reducirse o incluso desaparecer.

El análisis de los diferentes sectores sociales revela que, en el ámbito educativo, la disminución sostenida de la fecundidad llevó a grandes reducciones del cociente entre la población en edad de estudiar y aquella en edad de trabajar. Esto produjo una liberación de recursos financieros que posibilitaría una mayor inversión en la cobertura y calidad de la educación. Este hecho se observó en todos los países de la región y representó, en promedio, un $19 \%$ de aumento en los recursos disponibles por niño en edad escolar durante la última década. Del mismo modo, se prevé un aumento sustancial para el próximo decenio (del 15\% en promedio), lo que permitiría sostener económicamente los esfuerzos encaminados a ampliar la cobertura educativa en los niveles secundario y terciario. Un estudio reciente de la Cepal (2008b) señala justamente la oportunidad que se abre durante el periodo de bono 
demográfico en diversos países de la región para realizar fuertes inversiones en educación. El documento sugiere que aprovechar el bono demográfico a través de este tipo de inversiones, además de la creación de empleos productivos, no sólo implica mayores oportunidades para los jóvenes de hoy, sino que también representa una oportunidad única de prepararse para el futuro, ya que los logros económicos derivados de tales inversiones harán posible los saltos productivos y el ahorro necesarios para hacer frente al aumento exponencial de costos asociados al envejecimiento de la sociedad.

En el caso de los sistemas de salud, los países que se encuentran en las etapas menos avanzadas de la transición demográfica están en una situación particularmente favorable, ya que su población se concentra cada vez más en las edades económicamente activas, las que suelen hacer menos uso de los servicios médicos. Sin embargo, mientras en algunos países se prevé un aumento de los recursos disponibles para la salud por consumidor, resultante de los cambios en la estructura por edades, en aquellos más avanzados en la transición demográfica, donde la mayor proporción de la población se concentra cada vez más en las personas de edad, se proyecta más bien una desventaja demográfica. En Cuba, por ejemplo, se espera que los cambios en la estructura etaria redunden en una disminución del $5 \%$ de los recursos de salud disponibles por consumidor antes de 2018.

En gran contraste con el caso de la educación, el cambio demográfico impone mayores dificultades para el financiamiento de los sistemas de reparto de pensiones. El efecto del envejecimiento de la población en estos sistemas es evidente en casi todos

\section{Referencias}

ADIOETOMO, S. y otros. Policy implications of age-structural changes. CICRED Policy Papers Series, Paris, 2005.

BLOOM, D.; CANNING, D.; SEVILLA, J. The demographic dividend: a new perspective on the economic consequences of population change. RAND Population Matters Program, Santa Monica, California, № MR-1274, 2003. los países de la región. Durante la década pasada su costo se incrementó en un $9 \%$, debido a que el crecimiento de la población jubilada fue más rápido que el de aquella en edad de trabajar. Se espera que, en el próximo decenio, la carga de financiamiento de estos regímenes aumente, en promedio, un $17 \%$ por contribuyente. El impacto será especialmente sensible en los países más avanzados en la transición demográfica.

Efectivamente, el envejecimiento que se vislumbra en el futuro de cada uno de los países latinoamericanos resultará en una inversión de la ecuación de dependencia, con un aumento sostenido de dependientes mayores frente a jóvenes y adultos en edad de trabajar. Tal situación requerirá programas y políticas públicas de ajuste en diversas áreas, incluyendo la provisión de cuidados de larga duración y el financiamiento de pensiones a una población progresivamente envejecida. Turra y Queiroz (2008), por ejemplo, en su análisis del sistema de la seguridad social del Brasil, advierten la forma en que la falta de políticas adecuadas puede anular algunos de los beneficios temporales de los cambios demográficos y agravar los efectos adversos del envejecimiento de la población. Si se alcanza esa fase en condiciones económicas desfavorables, sin crecimiento y ahorro previos, la carga de la población dependiente sobre el grupo productivo exigirá grandes transferencias de recursos de las personas activas a las personas mayores dependientes, dando origen a una situación que puede generar no sólo conflictos intergeneracionales, sino también problemas de solvencia que podrían poner en riesgo el financiamiento de sistemas clave como la salud y la seguridad social.

Growth and the demographic transition. NBER Working Papers, National Bureau of Economic Research, vol. 6, 2006.

BLOOM, D.; WILLIAMSON, J.G. Demographic transitions and economic miracles in emerging Asia. The World Bank Economic Review, 12: 419-455; doi:10.1093/wber/12.3.419, 1998. 
CEPAL-Comisión Económica para América Latina y el Caribe. Transformaciones demográficas y su influencia en el desarrollo en América Latina y el Caribe. Santiago de Chile, Cepal, LC/ G.2378(SES.32/14), 2008a.

El bono demográfico: una oportunidad para avanzar en cobertura y progresión en educación secundaria (Capítulo III). Panorama social de América Latina 2008. Santiago de Chile, Cepal, LC/G.2402-P/E, 2008b.

Panorama social de América Latina 2006. Santiago de Chile, Cepal, LC/G.2326-P, 2006.

CEPAL/CELADE/BID -Comisión Económica para América Latina y el Caribe/Centro Latinoamericano de Demografía/Banco Interamericano de Desarrollo. Impacto de las tendencias demográficas sobre los sectores sociales en América Latina: contribución al diseño de políticas y programas. Serie E, Santiago de Chile, n. 45, LC/DEM/G.161, 1996.

FÍGOLI, M.G.B.; WONG, L.R. El camino hacia la estabilización demográfica y el proceso de envejecimiento en América Latina: una ilustración a partir de algunos países seleccionados. Papeles de Población, México, D.F., n. 35, 2003.

HARTIGAN, J.A.; WONG, M.A. A K-means clustering algorithm. Applied Statistics, v. 28, 1979.

LEE, R.; LEE, S.-H.; MASON, A. Charting the economic lifecycle: population aging, human capital accumulation, and productivity growth. Population and Development Review, Nueva York, Consejo de Población, Naciones Unidas, v. 33 (Supplement), 2007.

MASON, A. (Ed.). Population change and economic development in East Asia: challenges met, opportunities seized. Stanford University Press, 2002.
MASON, A. y otros. Population aging and intergenerational transfers: introducing age into national accounts. In: WISE, D.A. (Ed.). Developments in the economics of aging. University of Chicago Press, 2008.

TURRA, C. M.; QUEIROZ, B. L. Antes de que sea demasiado tarde: transición demográfica, mano de obra disponible y problemas de la seguridad social en el Brasil. Notas de Población, Santiago de Chile, Cepal, Nn. 86, p. 141-165, LC/ G.2349-P, 2009.

UNFPA - Fondo de Población de las Naciones Unidas. Shift to smaller families can bring economic benefits. News features, 1998. Disponible en: <http://www.unfpa.org/ swp/1998/newsfeature1.htm>. Accedido el 10 de junio de 2009.

WONG, L. R.; CARVALHO, J. A. Age-structural transition in Brazil: demographic bonuses and emerging challenges. In: POOL, I.; WONG, L.R. (Eds.). Age-structural transitions: challenges for development. París, Comité para la Cooperación Internacional en las Investigaciones Nacionales sobre Demografía (CICRED), 2006a.

.El rápido proceso de envejecimiento en los países del tercer mundo y las políticas sociales: el caso de Brasil. Notas de Población, Santiago de Chile, Cepal, n. 81, p. 1-31, LC/G.2300-P, 2006b.

WONG, L. R.; CARVALHO, J. A. M.; AGUIRRE, A. Duración de la transición demográfica en América Latina y su relación con el desarrollo humano. Estudios Demográficos y Urbanos, México D.F., El Colegio de México, n. 043, p. 185-207, 2000.

ZAVALA DE COSíO, M. E. Dos modelos de transición demográfica en América Latina.

Perfiles Latinoamericanos: revista de la Facultad Latinoamericana de Ciencias Sociales, Sede México, ISSN 0188-7653, $\mathrm{N}^{\circ} 6$, p. 29-47, 1995. 


\title{
Resumo
}

\section{Impacto das mudanças demográficas nas demandas setoriais na América Latina}

A América Latina está passando por um processo de transição demográfica que afeta profundamente a distribuição etária da população. Considerando que o comportamento econômico das pessoas varia conforme a etapa do ciclo da vida em que se encontram, as mudanças na estrutura etária podem vir a gerar um impacto relevante sobre o processo de desenvolvimento econômico. Com base nos padrões etários de consumo e renda, construídos a partir dos dados do projeto Cepal / IDRC sobre transferências intergeracionais, o artigo trata de examinar o impacto econômico das mudanças demográficas, em especial do bônus demográfico, sobre três setores diretamente relacionados com o bem-estar da população e o desenvolvimento econômico regional: educação, saúde e sistema de aposentadoria. As análises revelam que, na área da educação, está previsto um aumento substancial dos recursos disponíveis para a próxima década, o que permitiria sustentar economicamente os esforços de ampliação da cobertura nos diferentes níveis educativos. Na área da saúde, enquanto em alguns países se prevê um aumento dos recursos disponíveis por consumidor, nos mais avançados na transição demográfica, projeta-se uma desvantagem demográfica para o próximo decênio. Em grande contraste com a educação, a mudança demográfica gera maiores dificuldades para o financiamento dos sistemas de aposentadoria. Os efeitos do envelhecimento da população nestes sistemas estão patentes em quase todos os países da região

Palavras chave: Bônus demográfico. Envelhecimento. Setores sociais.

\begin{abstract}
Impact of demographic change on sectorial demands in Latin America

Latin America is undergoing a demographic transition that is transforming the age distribution of its population. Since economic behavior varies over one's life course, changes in age structures can generate significant impacts on processes of economic development. Using data on consumption patterns and income by age from the Cepal/IDRC Project on Intergenerational Transfers, this article examines the economic effect of demographic change, especially of demographic dividend, on three sectors directly related to the population's well-being and regional economic development: education, health and the retirement system. The analyses show that resources for education are likely to increase over the next decade, and this will probably sustain the current efforts at expanding the educational system at the various levels of schooling. In the area of health care, an increase in available resources per consumer can be expected in some countries, but, in countries where the demographic transition is farther advanced, a demographic change hinders expansion of health systems in the coming decades. Similarly, retirement systems will probably suffer strong negative impacts from changes in the demographic structure, since the effects of the aging of the population in these systems are evident in almost all the countries in the region.
\end{abstract}

Keywords: Demographic bonus. Aging. Social sectors.

Recebido para publicação em 23/03/2009. Aceito para publicação em 24/09/2009. 
